This is an Open Access article, distributed under the terms of the Creative Commons Attribution licence (http://creativecommons.org/licenses/by/4.0/), which permits unrestricted re-use, distribution, and reproduction in any medium, provided the original work is properly cited.

\title{
ALPS: the Arbitrary Linear Plasma Solver
}

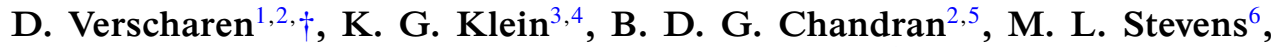 \\ C. S. Salem ${ }^{7}$ and S. D. Bale ${ }^{7,8}$ \\ ${ }^{1}$ Mullard Space Science Laboratory, University College London, Dorking RH5 6NT, UK \\ ${ }^{2}$ Space Science Center, University of New Hampshire, Durham, NH 03824, USA \\ ${ }^{3}$ Department of Climate and Space Sciences and Engineering, University of Michigan, Ann Arbor, \\ MI 48109, USA \\ ${ }^{4}$ Lunar and Planetary Laboratory, University of Arizona, Tucson, AZ 85719, USA \\ ${ }^{5}$ Department of Physics, University of New Hampshire, Durham, NH 03824, USA \\ ${ }^{6}$ Harvard Smithsonian Center for Astrophysics, Cambridge, MA 02138, USA \\ ${ }^{7}$ Space Sciences Laboratory, University of California, Berkeley, CA 94720, USA \\ ${ }^{8}$ Department of Physics, University of California, Berkeley, CA 94720, USA
}

(Received 13 March 2018; revised 19 June 2018; accepted 20 June 2018)

The Arbitrary Linear Plasma Solver (ALPS) is a parallelised numerical code that solves the dispersion relation in a hot (even relativistic) magnetised plasma with an arbitrary number of particle species with arbitrary gyrotropic equilibrium distribution functions for any direction of wave propagation with respect to the background field. ALPS reads the background momentum distributions as tables of values on a $\left(p_{\perp}, p_{\|}\right)$grid, where $p_{\perp}$ and $p_{\|}$are the momentum coordinates in the directions perpendicular and parallel to the background magnetic field, respectively. We present the mathematical and numerical approach used by ALPS and introduce our algorithms for the handling of poles and the analytic continuation for the Landau contour integral. We then show test calculations of dispersion relations for a selection of stable and unstable configurations in Maxwellian, bi-Maxwellian, $\kappa$-distributed and Jüttner-distributed plasmas. These tests demonstrate that ALPS derives reliable plasma dispersion relations. ALPS will make it possible to determine the properties of waves and instabilities in the non-equilibrium plasmas that are frequently found in space, laboratory experiments and numerical simulations.

Key words: plasma waves, plasma instabilities, space plasma physics

\section{Introduction}

The vast majority of the visible matter in the universe is in the plasma state. The solar wind is an example of such an astrophysical plasma. Due to its accessibility to spacecraft, it is the perfect environment for making comparisons between theoretical plasma-physics predictions and in situ observations in the astrophysical context with access to wide scale separations (see, for example, Marsch 2006). Plasmas can deviate

$\dagger$ Email address for correspondence: d.verscharen@ucl.ac.uk 
from thermodynamic equilibrium if the relaxation due to particle collisions occurs on time scales that are larger than the characteristic time scales of the collective plasma behaviour. Such a collisionless plasma is characterised by non-Maxwellian features in its velocity distribution functions. In the fast solar wind, this condition is frequently fulfilled, and, consequently, the observed distribution functions often deviate from the entropically favoured Maxwellian shape (Vasyliunas 1968; Gosling et al. 1981; Lui \& Krimigis 1981; Marsch et al. 1982a,b; Armstrong et al. 1983; Lui \& Krimigis 1983; Christon et al. 1988; Williams, Mitchell \& Christon 1988). In particular, beams and temperature anisotropies are some of the observed features in the distributions of ions and electrons in the solar wind (Pilipp et al. 1987a,b; Hellinger et al. 2006; Marsch 2006; Bale et al. 2009). If these deviations from equilibrium are suitably extreme, the plasma becomes unstable and generates waves or non-propagating structures that react back upon the plasma to reduce the deviations from equilibrium (Eviatar \& Schulz 1970; Schwartz 1980; Gary 1993; Hellinger \& Trávníček 2011, 2013). Also laboratory and fusion plasmas often show non-Maxwellian features in their velocity distribution functions. These include, for example, fast particles resulting from neutral-beam injections, alpha-particle beams from fusion reactions or non-Maxwellian distributions resulting from active ion/electron-cyclotron heating (Stix 1975; Gaffey 1976a,b; Tang 1978; Seki et al. 1989; Heidbrink \& Sadler 1994).

The behaviour of plasma waves and instabilities is typically studied with the help of numerical codes that solve the hot-plasma dispersion relation. Traditionally, these codes (like WHAMP, PLUME or NHDS) use a shifted bi-Maxwellian background distribution function as the zeroth-order description for the plasma state (Roennmark 1982; Quataert 1998; Klein et al. 2012; Verscharen \& Chandran 2018). For nearly collisionless plasmas, however, the bi-Maxwellian distribution function is a mathematical convenience rather than a reliable representation of the true plasma distribution function, and many space-plasma observations show that the bi-Maxwellian representation is not accurate (Hundhausen 1970; Leubner 1978; Marsch et al. 1982b; Pilipp et al. 1987a; Marsch \& Tu 2001; Štverák et al. 2009). Some previous approaches in non-Maxwellian solvers treated certain limits or geometries (Dum, Marsch \& Pilipp 1980; Summers \& Thorne 1991; Xue, Thorne \& Summers 1993; Summers, Xue \& Thorne 1994; Xue, Thorne \& Summers 1996; Hellberg, Mace \& Cattaert 2005; Cattaert, Hellberg \& Mace 2007; Lazar \& Poedts 2009; Mace \& Sydora 2010; Lazar, Poedts \& Schlickeiser 2011; Galvaõ et al. 2012; Xie 2013; Lazar \& Poedts 2014; Gaelzer \& Ziebell 2016; Gaelzer, Ziebell \& Meneses 2016) or faced challenges in the weakly damped limit (Hellinger \& Trávníček 2011).

We present our numerical code ALPS (Arbitrary Linear Plasma Solver), which solves the full hot-plasma dispersion relation in a plasma consisting of an arbitrary number of particle species with arbitrary background distribution functions $f_{0 j}$ and with arbitrary directions of wave propagation with respect to the uniform background magnetic field. ALPS is also able to solve the dispersion relation for relativistic plasmas. Matsuda \& Smith (1992) developed a code similar to ALPS that calculates the dispersion relation in an arbitrary plasma with relativistic effects. Their code uses a cubic-spline fit to both fill data gaps and approximate the analytic continuation, while ALPS uses a novel method called hybrid analytic continuation. The spline method forfeits its accuracy for strongly damped solutions since the calculation of the dispersion relation requires the evaluation of the spline at a complex value that is distant from the real grid points by which the spline is supported. Our method does not suffer from this problem. Astfalk \& Jenko (2017) also use a cubic-spline interpolation for the analytic continuation and as the basis for the integration in their 
code LEOPARD. This procedure allows for algebraic simplifications that enhance the speed of the integration significantly. LEOPARD, however, does not capture relativistic effects.

In $\S 2$, we review the underlying theory of the hot-plasma dispersion relation. $\S 3$ presents ALPS's numerical approach. In $\S 4$, we compare ALPS results to known limits of the hot-plasma dispersion relation such as Maxwellian, bi-Maxwellian, $\kappa$-distributed, and relativistic pair plasmas. In $\S 5$, we discuss our results and the applicability of ALPS to measured plasma distributions. The appendices describe how ALPS solutions depend on the resolution of the background distributions, discuss the Levenberg-Marquardt-fit routine used in our hybrid analytic continuation method and describe our strategy for numerically refining coarse-grained distribution functions obtained from spacecraft measurements.

\section{The linear dispersion relation of a hot plasma}

In this section, we discuss the mathematical basis for the calculation of the hot-plasma dispersion relation following the presentation and notation of Stix (1992). The determination of the kinetic wave dispersion relation in a hot plasma is based on the linearised set of Maxwell's equations and the linearised Vlasov equation (Trubnikov 1959; Drummond \& Rosenbluth 1963; Shkarofsky 1966; Stix 1992; Gary 1993). A wave or instability is then associated with a first-order perturbation $\delta f_{j}$ in the distribution function of species $j$ about a prescribed time-averaged background distribution function $f_{0 j}$,

$$
f_{j}(\boldsymbol{r}, \boldsymbol{p}, t)=f_{0 j}(\boldsymbol{p})+\delta f_{j}(\boldsymbol{r}, \boldsymbol{p}, t)
$$

where $\boldsymbol{r}$ is the spatial coordinate and $\boldsymbol{p}$ is the momentum coordinate. As with the distribution function $f_{j}$ in (2.1), we take the magnetic field $\boldsymbol{B}$ to be the sum of a uniform background magnetic field $\boldsymbol{B}_{0}$ and a fluctuating magnetic field $\delta \boldsymbol{B}$. We assume that $\boldsymbol{E}=\delta \boldsymbol{E}$; i.e. the average electric field is zero. Linear theory expresses $\delta f_{j}$ as a function of $f_{0 j}$ and the electromagnetic field components.

The distribution function $f_{j}$ in a collisionless plasma evolves according to the Vlasov equation,

$$
\frac{\partial f_{j}}{\partial t}+\boldsymbol{v} \cdot \frac{\partial f_{j}}{\partial \boldsymbol{r}}+q_{j}\left(\boldsymbol{E}+\frac{\boldsymbol{v}}{c} \times \boldsymbol{B}\right) \cdot \frac{\partial f_{j}}{\partial \boldsymbol{p}}=0,
$$

where $q_{j}$ is the charge of a particle of species $j, c$ is the speed of light and $\boldsymbol{v}$ is the velocity coordinate. We assume that all fluctuating quantities behave like plane waves; i.e. $\propto \exp (\mathrm{i} \boldsymbol{k} \cdot \boldsymbol{r}-\mathrm{i} \omega t)$, where $\boldsymbol{k}$ is the wave vector and $\omega$ is the (complex) frequency. Linearising equation (2.2), using Faraday's law and applying the method of characteristics, we obtain

$$
\begin{aligned}
\delta f_{j}= & -q_{j} \mathrm{e}^{\mathrm{i} k \cdot r-\mathrm{i} \omega t} \int_{0}^{\infty} \mathrm{d} \tau \mathrm{e}^{\mathrm{i} \alpha}\left\{E_{x} U \cos \left(\phi+\Omega_{j} \tau\right)+E_{y} U \sin \left(\phi+\Omega_{j} \tau\right)\right. \\
& \left.+E_{z}\left[\frac{\partial f_{0 j}}{\partial p_{\|}}-V \cos \left(\phi-\vartheta+\Omega_{j} \tau\right)\right]\right\},
\end{aligned}
$$

where $\boldsymbol{E}=\left(E_{x}, E_{y}, E_{z}\right)$ is the electric field, $\phi$ is the azimuthal angle of the momentum vector $\boldsymbol{p}, \vartheta$ is the azimuthal angle of the wave vector $\boldsymbol{k}$, the index $\perp(\|)$ refers to the 
direction perpendicular (parallel) with respect to the background magnetic field $\boldsymbol{B}_{0}$,

$$
\Omega_{j} \equiv \frac{q_{j} B_{0}}{m_{j} c \sqrt{1+\left(p_{\perp}^{2}+p_{\|}^{2}\right) / m_{j}^{2} c^{2}}}
$$

is the relativistic gyrofrequency, $m_{j}$ is the rest mass of a particle of species $j$,

$$
\begin{gathered}
\alpha \equiv-\frac{k_{\perp} v_{\perp}}{\Omega_{j}}\left[\sin \left(\phi-\vartheta+\Omega_{j} \tau\right)-\sin (\phi-\vartheta)\right]+\left(\omega-k_{\|} v_{\|}\right) \tau, \\
U \equiv \frac{\partial f_{0 j}}{\partial p_{\perp}}+\frac{k_{\|}}{\omega}\left(v_{\perp} \frac{\partial f_{0 j}}{\partial p_{\|}}-v_{\|} \frac{\partial f_{0 j}}{\partial p_{\perp}}\right)
\end{gathered}
$$

and

$$
V \equiv \frac{k_{\perp}}{\omega}\left(v_{\perp} \frac{\partial f_{0 j}}{\partial p_{\|}}-v_{\|} \frac{\partial f_{0 j}}{\partial p_{\perp}}\right) .
$$

We normalise $f_{j}$ so that $\int \mathrm{d}^{3} \boldsymbol{p} f_{0 j}=1$. The first velocity moments of the distribution functions of all species define the current density $j$ through

$$
\boldsymbol{j}=\sum_{j} q_{j} n_{0 j} \int \mathrm{d}^{3} \boldsymbol{p} \boldsymbol{v} \delta f_{j}=-\frac{\mathrm{i} \omega}{4 \pi} \sum_{j} \chi_{j} \cdot \boldsymbol{E},
$$

where $\chi_{j}$ is the contribution of species $j$ to the plasma susceptibility and $n_{0 j}$ is the background density of species $j$. Without loss of generality, we choose a cylindrical coordinate system in which $k_{y}=\vartheta=0$ and apply a set of Bessel-function identities in order to facilitate the integration over $\phi$ and $\tau$ in (2.3). This allows us to rewrite the plasma susceptibilities as (provided that $\operatorname{Im}(\omega)>0$ )

$$
\begin{aligned}
\chi_{j}= & \frac{\omega_{\mathrm{p} j}^{2}}{\omega \Omega_{0 j}} \int_{0}^{\infty} 2 \pi p_{\perp} \mathrm{d} p_{\perp} \int_{-\infty}^{+\infty} \mathrm{d} p_{\|}\left[\hat{\boldsymbol{e}}_{\|} \hat{\boldsymbol{e}}_{\|} \frac{\Omega_{j}}{\omega}\left(\frac{1}{p_{\|}} \frac{\partial f_{0 j}}{\partial p_{\|}}-\frac{1}{p_{\perp}} \frac{\partial f_{0 j}}{\partial p_{\perp}}\right) p_{\|}^{2}\right. \\
& \left.+\sum_{n=-\infty}^{+\infty} \frac{\Omega_{j} p_{\perp} U}{\omega-k_{\|} v_{\|}-n \Omega_{j}} \boldsymbol{T}_{n}\right]
\end{aligned}
$$

where $\omega_{\mathrm{p} j} \equiv \sqrt{4 \pi n_{0 j} q_{j}^{2} / m_{j}}$ is the plasma frequency of species $j, \Omega_{0 j} \equiv q_{j} B_{0} / m_{j} c$ is the non-relativistic gyrofrequency and the tensor $\boldsymbol{T}_{n}$ is defined as

$$
\boldsymbol{T}_{n} \equiv\left(\begin{array}{ccc}
\frac{n^{2} \mathrm{~J}_{n}^{2}}{z^{2}} & \frac{\mathrm{i} n \mathrm{~J}_{n} \mathrm{~J}_{n}^{\prime}}{z} & \frac{n \mathrm{~J}_{n}^{2} p_{\|}}{z p_{\perp}} \\
-\frac{\mathrm{i} n \mathrm{~J}_{n} \mathrm{~J}_{n}^{\prime}}{z} & \left(\mathrm{~J}_{n}^{\prime}\right)^{2} & -\frac{\mathrm{iJ}_{n} \mathrm{~J}_{n}^{\prime} p_{\|}}{p_{\perp}} \\
\frac{n \mathrm{~J}_{n}^{2} p_{\|}}{z p_{\perp}} & \frac{\mathrm{iJ}_{n} \mathrm{~J}_{n}^{\prime} p_{\|}}{p_{\perp}} & \frac{\mathrm{J}_{n}^{2} p_{\|}^{2}}{p_{\perp}^{2}}
\end{array}\right),
$$

where $z \equiv k_{\perp} v_{\perp} / \Omega_{j}$ and $\mathrm{J}_{n} \equiv \mathrm{J}_{n}(z)$ is the $n$ th-order Bessel function. For $\operatorname{Im}(\omega) \leqslant 0$, the integral over $p_{\|}$is executed as the Landau integral after analytic continuation 
(for details, see chapter 8 of Stix 1992). Equation (2.9) describes the susceptibility for a general background distribution function $f_{0 j}$ in a relativistic plasma. The only assumptions are gyrotropy in $f_{0 j}$ and small amplitudes in the fluctuations so that linearisation is applicable, and a uniform, stationary equilibrium. The numerical challenge in the solution of the plasma dispersion relation results from the integrals over $p_{\perp}$ and $p_{\|}$in (2.9). We note that, in numerous classical codes for calculation of the linear hot-plasma dispersion relation (Roennmark 1982; Gary 1993; Klein \& Howes 2015; Verscharen \& Chandran 2018), these integrals are greatly simplified by assuming that $f_{0 j}$ is a (bi)-Maxwellian.

The dielectric tensor $\boldsymbol{\varepsilon}$ of the plasma is related to the plasma susceptibilities from (2.9) through

$$
\boldsymbol{\varepsilon}=\mathbf{1}+\sum_{j} \chi_{j}
$$

Finally, combining Faraday's law and Ampère's law leads to the wave equation,

$$
\boldsymbol{n}_{\mathrm{r}} \times\left(\boldsymbol{n}_{\mathrm{r}} \times \boldsymbol{E}\right)+\boldsymbol{\varepsilon} \cdot \boldsymbol{E} \equiv \mathcal{D} \cdot \boldsymbol{E}=0,
$$

where $\boldsymbol{n}_{\mathrm{r}} \equiv \boldsymbol{k} c / \omega$ is the refractive index. By setting $\operatorname{det} \mathcal{D}=0$, we obtain the dispersion relations $\omega=\omega(\boldsymbol{k})$ for non-trivial solutions to (2.12). We write these solutions in the form $\omega=\omega_{\mathrm{r}}+\mathrm{i} \gamma$, where $\omega_{\mathrm{r}}=\operatorname{Re}(\omega)$ and $\gamma=\operatorname{Im}(\omega)$.

\section{Numerical approach}

In order to find the solutions to the hot-plasma dispersion relation, ALPS determines the values of $\omega_{\mathrm{r}}$ and $\gamma$ that solve (2.12) for specified background distributions $f_{0 j}$ at a given set of values for $\boldsymbol{k}, m_{j}, q_{j}, n_{0 j}$ and $v_{\mathrm{A}} / c$, where $v_{\mathrm{A}} \equiv B_{0} / \sqrt{4 \pi n_{0 \mathrm{p}} m_{\mathrm{p}}}$. ALPS uses an efficient iterative Newton-secant algorithm to solve (2.12) based on an initial guess for $\omega_{\mathrm{r}}$ and $\gamma$ (Press et al. 1992). The numerically challenging part for this calculation is the evaluation of $\chi_{j}$ in (2.9). In the following, we present ALPS's strategy for this evaluation in the non-relativistic case. We discuss the extension to relativistic cases with poles in the integration domain in $\S 3.3$, which is equivalent to the non-relativistic case with the exception that the coordinate system is transformed from $\left(p_{\perp}, p_{\|}\right)$to $\left(\Gamma, \bar{p}_{\|}\right)$, where $\Gamma$ is the Lorentz factor and $\bar{p}_{\|} \equiv p_{\|} / m_{j} c$, and that (3.16) below is used instead of (2.9).

We prescribe the shape of $f_{0 j}$ in input files for each species (called ' $f_{0}$ table') as an ASCII table that lists $p_{\perp}, p_{\|}$and the associated values of $f_{0 j}$. From this table, we calculate $\partial f_{0 j} / \partial p_{\perp}$ and $\partial f_{0 j} / \partial p_{\|}$on the same grid as the $f_{0}$ table using second-order finite differencing. We do not include the derivatives at the outer boundaries of the $\left(p_{\perp}, p_{\|}\right)$domain. The resolution of the $f_{0}$ table is given by $N_{\perp}$ points in the $p_{\perp}$ direction and $N_{\|}$points in the $p_{\|}$-direction. The table spans from $p_{\perp}=0$ to $p_{\perp}=P_{\max , \perp j}$ in the perpendicular direction and from $p_{\|}=-P_{\max , \| j}$ to $p_{\|}=P_{\max , \| j}$ in the parallel direction.

The integration in (2.9) allows us to integrate separately and independently for each $n$ and $j$. This provides us with a very natural way to parallelise the calculation scheme by assigning the separate integrations to different processors. We use MPI for the parallelisation. The integrating nodes return their contributions to $\chi_{j}$ to the master node, which then sums up the contributions, determines the value of $\boldsymbol{\varepsilon}$ and updates the values of $\omega_{\mathrm{r}}$ and $\gamma$ through a Newton-secant step. The updated values for $\omega_{\mathrm{r}}$ and $\gamma$ are then returned to the integrating nodes, which afterwards evaluate the integration 
of their updated contribution to $\chi_{j}$. We evaluate all values of $n$ up to a value of $\pm n_{\max }$, which is determined as the value of $n$ for which the maximum value of $\left|\mathbf{J}_{n}\right|$ is smaller than the user-defined parameter $J_{\max }$. The necessary value of $n_{\max }$ depends on the wavenumber, the direction of propagation of the treated wave and the thermal speeds of the plasma components. In bi-Maxwellian codes under typical solar-wind conditions, the accuracy of the dispersion relation is better than $\Delta|\omega| /|\omega| \sim 10^{-5}$ for $J_{\max } \sim 10^{-45}$ (which typically corresponds to $n_{\max } \gtrsim 10$ at proton scales).

We use a standard two-dimensional trapezoidal integration scheme to integrate over $p_{\perp}$ and $p_{\|}$. However, this scheme breaks down near the poles of the integrand in (2.9) and requires a special treatment of the analytic continuation when $\gamma \leqslant 0$. In the remainder of this section, we discuss our strategies to resolve these numerical difficulties.

\subsection{Integrating near poles}

A challenge concerning the numerical integration is the treatment of the poles that occur in the term proportional to $\boldsymbol{T}_{n}$ in (2.9). The integrals in question are of the form

$$
I\left(p_{\perp}\right) \equiv \int_{-\infty}^{+\infty} \mathrm{d} p_{\|} \frac{\Omega_{j} U \boldsymbol{T}_{n}}{\omega-k_{\|} v_{\|}-n \Omega_{j}} \equiv \int_{-\infty}^{+\infty} \mathrm{d} p_{\|} G\left(p_{\perp}, p_{\|}\right)
$$

for $\gamma>0$. For sufficiently small $\gamma$, the denominator in (3.1) can become very small along the real $p_{\|}$axis so that the grid sampling leads to large numerical errors in the integration. To describe how we evaluate these integrals, we first rewrite the integral in (3.1) in the more generic form

$$
\mathcal{I} \equiv \int_{-\infty}^{+\infty} \mathrm{d} x \frac{g(x)}{x-t_{\mathrm{r}}-\mathrm{i} t_{\mathrm{i}}}
$$

where $x, t_{\mathrm{r}}$ and $t_{\mathrm{i}}$ are real, $g(x)$ is a smooth function and the integration is performed along the real axis. We choose a symmetric interval $\left[t_{\mathrm{r}}-\Delta, t_{\mathrm{r}}+\Delta\right]$ around $t_{\mathrm{r}}$ where $\Delta \ll g\left(t_{\mathrm{r}}\right) / g^{\prime}\left(t_{\mathrm{r}}\right)$, and write

$$
\mathcal{I}=\int_{t_{\mathrm{r}}-\Delta}^{t_{\mathrm{r}}-\Delta} \mathrm{d} x \frac{g(x)}{x-t_{\mathrm{r}}-\mathrm{i} t_{\mathrm{i}}}+\text { rest }
$$

where 'rest' refers to the integration outside the interval $\left[t_{\mathrm{r}}-\Delta, t_{\mathrm{r}}+\Delta\right]$. We define a function $f(x)$ to be odd with respect to $t_{\mathrm{r}}$ if $f(x)=-f\left(2 t_{\mathrm{r}}-x\right)$, and even with respect to $t_{\mathrm{r}}$ if $f(x)=f\left(2 t_{\mathrm{r}}-x\right)$. Following Longman (1958) and Davis \& Rabinowitz (1984), we then separate the integrand into its odd and even parts with respect to $t_{\mathrm{r}}$ as

$$
\begin{aligned}
\mathcal{I}= & \frac{1}{2} \int_{t_{\mathrm{r}}-\Delta}^{t_{\mathrm{r}}+\Delta} \mathrm{d} x\left[\frac{g(x)}{x-t_{\mathrm{r}}-\mathrm{i} t_{\mathrm{i}}}-\frac{g\left(2 t_{\mathrm{r}}-x\right)}{-x+t_{\mathrm{r}}-\mathrm{i} t_{\mathrm{i}}}\right] \\
& +\frac{1}{2} \int_{t_{\mathrm{r}}-\Delta}^{t_{\mathrm{r}}+\Delta} \mathrm{d} x\left[\frac{g(x)}{x-t_{\mathrm{r}}-\mathrm{i} t_{\mathrm{i}}}+\frac{g\left(2 t_{\mathrm{r}}-x\right)}{-x+t_{\mathrm{r}}-\mathrm{i} t_{\mathrm{i}}}\right]+\text { rest. }
\end{aligned}
$$

The integrand in the first integral in (3.4) is odd with respect to $t_{\mathrm{r}}$ and thus vanishes after the integration over the symmetric interval around $t_{\mathrm{r}}$. The second integral, on the other hand, is even with respect to $t_{\mathrm{r}}$ and thus

$$
\mathcal{I}=\int_{t_{\mathrm{r}}}^{t_{\mathrm{r}}+\Delta} \mathrm{d} x\left[\frac{g(x)}{x-t_{\mathrm{r}}-\mathrm{i} t_{\mathrm{i}}}-\frac{g\left(2 t_{\mathrm{r}}-x\right)}{x-t_{\mathrm{r}}+\mathrm{i} t_{\mathrm{i}}}\right]+\text { rest. }
$$


We define $\Delta$ through a user-defined parameter $M_{\mathrm{I}}$ so that $\Delta \equiv M_{\mathrm{I}} \Delta p_{\|}$, where $\Delta p_{\|}$is the size of a grid step in the parallel direction. We then define $\delta \equiv \Delta / M_{\mathrm{P}}$, where $M_{\mathrm{P}}$ is another user-defined parameter. Except for cases in which $\left|t_{\mathrm{i}}\right|$ is extremely small, we apply a trapezoidal integration over $M_{\mathrm{P}}$ steps of width $\delta$ to the integral in (3.5). The smoothness of $g(x)$ allows us to expand $g(x)$ around the nearest grid point of the $M_{\mathrm{I}}$ grid points in the interval $\left[t_{\mathrm{r}}, t_{\mathrm{r}}+\Delta\right]$ using a Taylor series. By taking $\Delta p_{\|}$to be sufficiently small, we can retain just the first two terms in the series without losing significant accuracy. Since the integral in (3.5) does not converge numerically if $\left|t_{\mathrm{i}}\right|$ is extremely small, we implement the following procedure when $\left|t_{\mathrm{i}}\right| \leqslant t_{\text {lim }}$, where $t_{\text {lim }}$ is a user-defined parameter. We first rewrite (3.5) using truncated Taylor expansions of $g(x)$ and $g\left(2 t_{\mathrm{r}}-x\right)$ around $x=t_{\mathrm{r}}$ as

$$
\mathcal{I}=\int_{t_{\mathrm{r}}}^{t_{\mathrm{r}}+\Delta} \mathrm{d} x\left[\frac{2 \mathrm{i} t_{\mathrm{i}} g\left(t_{\mathrm{r}}\right)}{\left(x-t_{\mathrm{r}}\right)^{2}+t_{\mathrm{i}}^{2}}+\frac{2 g^{\prime}\left(t_{\mathrm{r}}\right)\left(x-t_{\mathrm{r}}\right)^{2}}{\left(x-t_{\mathrm{r}}\right)^{2}+t_{\mathrm{i}}^{2}}\right]+\text { rest. }
$$

We determine $g\left(t_{\mathrm{r}}\right)$ and $g^{\prime}\left(t_{\mathrm{r}}\right)$ through linear interpolation between the neighbouring grid points to $t_{\mathrm{r}}$. The term proportional to $g^{\prime}\left(t_{\mathrm{r}}\right)$ in (3.6) converges numerically for any value of $t_{\mathrm{i}}$. We set the term proportional to $g\left(t_{\mathrm{r}}\right)$ equal to its small- $t_{\mathrm{i}}$ limit, namely

$$
\int_{t_{\mathrm{r}}}^{t_{\mathrm{r}}+\Delta} \mathrm{d} x \frac{2 \mathrm{i} t_{\mathrm{i}} g\left(t_{\mathrm{r}}\right)}{\left(x-t_{\mathrm{r}}\right)^{2}+t_{\mathrm{i}}^{2}}=\mathrm{i} \pi g\left(t_{\mathrm{r}}\right) \operatorname{sgn}\left(t_{\mathrm{i}}\right) .
$$

We use this method for both the integration of $\chi_{j}$ near poles and the principal-value integration that is necessary if $\gamma=0$.

\subsection{Analytic continuation}

If $\gamma \leqslant 0$, the integration in (2.9) requires an analytic continuation into the complex plane. If $f_{0 j}$ were given as a closed algebraic expression, the analytic continuation would simply entail the evaluation of $f_{0 j}\left(p_{\perp}, p_{\|}\right)$at a complex value for $p_{\|}$in the non-relativistic case. In our case, however, $f_{0 j}$ is only defined on a real grid in $p_{\perp}$ and $p_{\|}$, yet the analytic continuation of $f_{0 j}$ is still uniquely defined. This leads to the known mathematical problem of numerical analytic continuation (Cannon \& Miller 1965; Reichel 1986; Fujiwara et al. 2007; Fu et al. 2012; Zhang \& Ma 2013; Kranich 2014). Our solution for this problem is our hybrid analytic continuation scheme. We note that this approach is only relevant for damped modes, i.e. $\gamma \leqslant 0$.

Landau's rule of integration around singularities (Landau 1946; Lifshitz \& Pitaevskii 1981) leads to the following three cases with the appropriate residues for the evaluation of $I\left(p_{\perp}\right)$ for general $\gamma$ :

$$
I\left(p_{\perp}\right)=\int_{C_{\mathrm{L}}} \mathrm{d} p_{\|} G\left(p_{\perp}, p_{\|}\right)= \begin{cases}\int_{-\infty}^{+\infty} \mathrm{d} p_{\|} G\left(p_{\perp}, p_{\|}\right) & \text {if } \gamma>0, \\ \mathcal{P} \int_{-\infty}^{+\infty} \mathrm{d} p_{\|} G\left(p_{\perp}, p_{\|}\right)+\mathrm{i} \pi \sum \operatorname{Res}_{A}(G) & \text { if } \gamma=0, \\ \int_{-\infty}^{+\infty} \mathrm{d} p_{\|} G\left(p_{\perp}, p_{\|}\right)+2 \mathrm{i} \pi \sum \operatorname{Res}_{A}(G) & \text { if } \gamma<0,\end{cases}
$$

where $C_{\mathrm{L}}$ is the contour of the Landau integration, which lies below the complex poles in the integrand. The integrations on the right-hand side of (3.8) are performed along 
the real axis, and $\mathcal{P}$ indicates the principal-value integral. The sum sign indicates the summation over the residues of all poles $A$ of the function $G$. In a non-relativistic plasma, $G$ has one simple pole, and thus

$$
\sum \operatorname{Res}_{A}(G)=-\left.\frac{m_{j}}{\left|k_{\|}\right|} \Omega_{j} U \boldsymbol{T}_{n}\right|_{p_{\|}=p_{\text {pole }}},
$$

where $p_{\text {pole }}=m_{j}\left(\omega-n \Omega_{j}\right) / k_{\|}$is the parallel momentum associated with pole $A$.

It is a common approach to decompose the background distribution functions in terms of analytical expressions and then to evaluate these at the complex poles. Complete orthogonal basis functions such as Hermite, Legendre or Chebyshev polynomials are the prime candidates for such a decomposition since they can represent $f_{0 j}$ to an arbitrary degree of accuracy (Robinson 1990; Weideman 1995; Xie 2013). These approaches are useful when $f_{0 j}$ deviates only slightly from a Maxwellian. They require, however, very high orders of decomposition and are thus slow in the presence of typical structures that we see in the solar wind such as a proton core-beam configuration. Therefore, they are unsuitable for ALPS's purpose, and we pursue a different approach, which we call the hybrid analytic continuation. The basic idea behind this approach is to integrate $I$ numerically along the real axis whenever possible and to resort to an algebraic function for the sole purpose of the evaluation of $\operatorname{Res}_{A}(G)$ when necessary.

For the determination of an appropriate algebraic function, ALPS allows the user to choose an arbitrary combination of fit functions to represent $f_{0 j}$ and automatically evaluates the fits before the integration begins. The code evaluates the fits separately at each $p_{\perp}$, so that no assumption is made as to the structure of $f_{0 j}$ in the $p_{\perp}$-direction. ALPS uses these functions only if a pole is within the integration domain and only if $\gamma \leqslant 0$. The intrinsic fit functions that the code can combine include a Maxwellian distribution,

$$
f_{0 j}=\frac{1}{\pi^{3 / 2} m_{j}^{3} w_{\perp j}^{2} w_{\|}} \exp \left(-\frac{p_{\perp}^{2}}{m_{j}^{2} w_{\perp j}^{2}}-\frac{\left(p_{\|}-m_{j} U_{j}\right)^{2}}{m_{j}^{2} w_{\| j}^{2}}\right),
$$

where $w_{\perp j} \equiv \sqrt{2 k_{\mathrm{B}} T_{\perp j} / m_{j}}\left(w_{\| j} \equiv \sqrt{2 k_{\mathrm{B}} T_{\| j} / m_{j}}\right)$ is the thermal speed of species $j$ in the direction perpendicular (parallel) with respect to $\boldsymbol{B}_{0}, T_{\perp j}\left(T_{\| j}\right)$ is the temperature of species $j$ perpendicular (parallel) to $\boldsymbol{B}_{0}, k_{\mathrm{B}}$ is the Boltzmann constant and $U_{j}$ is the $\boldsymbol{B}_{0}$-parallel drift speed of species $j$; a $\kappa$-distribution (Summers et al. 1994; Astfalk, Görler \& Jenko 2015),

$$
\begin{aligned}
f_{0 j}= & \frac{1}{m_{j}^{3} w_{\perp j}^{2} w_{\| j}}\left[\frac{2}{\pi(2 \kappa-3)}\right]^{3 / 2} \frac{\tilde{\Gamma}(\kappa+1)}{\tilde{\Gamma}(\kappa-1 / 2)} \\
& \times\left\{1+\frac{2}{2 \kappa-3}\left[\frac{p_{\perp}^{2}}{m_{j}^{2} w_{\perp j}^{2}}+\frac{\left(p_{\|}-m_{j} U_{j}\right)^{2}}{m_{j}^{2} w_{\| j}^{2}}\right]\right\}^{-(\kappa+1)} ;
\end{aligned}
$$

and a Jüttner distribution (Jüttner 1911; Chacón-Acosta, Dagdug \& Morales-Técotl 2010),

$$
f_{0 j}=\frac{1}{2 \pi m_{j}^{3} c w_{j}^{2} \mathrm{~K}_{2}\left(2 c^{2} / w_{j}^{2}\right)} \exp \left(-2 \frac{c^{2}}{w_{j}^{2}} \sqrt{1+\frac{|\boldsymbol{p}|^{2}}{m_{j}^{2} c^{2}}}\right) ;
$$

where $\kappa$ is the $\kappa$-index and $\mathrm{K}_{2}$ is the modified Bessel function of the second kind. The Jüttner distribution is the thermodynamic equilibrium distribution if $k_{\mathrm{B}} T_{j} \gtrsim m_{j} c^{2}$. 
The exponential in (3.12) reduces to the Maxwellian $\exp \left(-v^{2} / w_{j}^{2}\right)$ with a different $\boldsymbol{p}$-independent normalisation factor for $p^{2} / m_{j}^{2} c^{2} \ll 1$. We use an automated LevenbergMarquardt-fit algorithm (Levenberg 1944; Marquardt 1963) and describe the details of the fit routine in appendix B.

\subsection{The poles in a relativistic plasma}

The analytic continuation and pole handling in the relativistic case entail a further complication due to the non-trivial $\boldsymbol{p}$-dependence of the resonant denominator in (2.9) (Buti 1962; Lerche 1968). We define a plasma to be relativistic when there is a significant number of particles at relativistic velocities. This can be the case in plasmas with relativistic temperatures $\left(k_{\mathrm{B}} T_{j} \gtrsim m_{j} c^{2}\right)$ or in plasmas with relativistic beams $\left(P_{j} \gtrsim m_{j} c\right.$, where $P_{j}$ is the drift momentum). Using the relativistic expression for $\Omega_{j}$ in (2.4) shows that we can write for the pole of the function under the integral sign in (3.1)

$$
\frac{1}{\omega-k_{\|} v_{\|}-n \Omega_{j}}=-\frac{1}{k_{\|}} \frac{\Gamma m_{j}}{\left(p_{\|}-\frac{\omega}{k_{\|}} \Gamma m_{j}+n \frac{\Omega_{0 j}}{k_{\|}} m_{j}\right)},
$$

where

$$
\Gamma \equiv \sqrt{1+\frac{p_{\perp}^{2}+p_{\|}^{2}}{m_{j}^{2} c^{2}}}
$$

is the Lorentz factor. We define the dimensionless parallel momentum $\bar{p}_{\|} \equiv p_{\|} / m_{j} c$. The dimensionless parallel momentum associated with the relativistic pole is given by

$$
\bar{p}_{\text {pole }}=\Gamma \frac{\omega}{k_{\|} c}-\frac{n \Omega_{0 j}}{k_{\|} c} .
$$

We apply the technique proposed by Lerche (1967) to transform (2.9) from the $\left(p_{\perp}, p_{\|}\right)$coordinate system to the $\left(\Gamma, \bar{p}_{\|}\right)$coordinate system (see also Swanson 2002; Lazar \& Schlickeiser 2006; López et al. 2014, 2016). This transformation yields

$$
\begin{aligned}
\chi_{j}= & 2 \pi m_{j}^{3} c^{3} \frac{\omega_{\mathrm{p} j}^{2}}{\omega \Omega_{0 j}} \int_{1}^{\infty} \mathrm{d} \Gamma \int_{-\sqrt{\Gamma^{2}-1}}^{+\sqrt{\Gamma^{2}-1}} \mathrm{~d} \bar{p}_{\|}\left[\hat{\boldsymbol{e}}_{\|} \hat{\boldsymbol{e}}_{\|} \frac{\Omega_{0 j}}{\omega} \bar{p}_{\|} \frac{\partial f_{0 j}}{\partial \bar{p}_{\|}}\right. \\
& \left.-\sum_{n=-\infty}^{+\infty} \frac{\Omega_{0 j}}{k_{\|} c}\left(\frac{\partial f_{0 j}}{\partial \Gamma}+\frac{k_{\|} c}{\omega} \frac{\partial f_{0 j}}{\partial \bar{p}_{\|}}\right) \frac{1}{\bar{p}_{\|}-\Gamma \frac{\omega}{k_{\|} c}+\frac{n \Omega_{0 j}}{k_{\|} c}} \overline{\boldsymbol{T}}_{n}\right],
\end{aligned}
$$

where

$$
\overline{\boldsymbol{T}}_{n} \equiv\left(\begin{array}{ccc}
\frac{n^{2} \mathrm{~J}_{n}^{2}}{\bar{z}^{2}} & \frac{\mathrm{i} n \mathrm{~J}_{n} \mathrm{~J}_{n}^{\prime}}{\bar{z}} \bar{p}_{\perp} & \frac{n \mathrm{~J}_{n}^{2} \bar{p}_{\|}}{\bar{z}} \\
-\frac{\mathrm{i} n \mathrm{~J}_{n} \mathrm{~J}_{n}^{\prime}}{\bar{z}} \bar{p}_{\perp} & \left(\mathrm{J}_{n}^{\prime}\right)^{2} \bar{p}_{\perp}^{2} & -\mathrm{i}_{n} \mathrm{~J}_{n}^{\prime} \bar{p}_{\|} \bar{p}_{\perp} \\
\frac{n \mathrm{~J}_{n}^{2} \bar{p}_{\|}}{\bar{z}} & \mathrm{iJ}_{n} \mathrm{~J}_{n}^{\prime} \bar{p}_{\|} \bar{p}_{\perp} & \mathrm{J}_{n}^{2} \bar{p}_{\|}^{2}
\end{array}\right),
$$


$\bar{p}_{\perp} \equiv \sqrt{\Gamma^{2}-1-\bar{p}_{\|}^{2}}, \bar{z} \equiv k_{\perp} c / \Omega_{0 j}$ and the Bessel functions are evaluated as $\mathrm{J}_{n} \equiv \mathrm{J}_{n}\left(\bar{z} \bar{p}_{\perp}\right)$. Whenever ALPS performs a relativistic calculation and

$$
-P_{\max , \| j} \leqslant \operatorname{Re}\left(\bar{p}_{\text {pole }}\right) \leqslant+P_{\max , \| j}
$$

the code automatically transforms from $\left(p_{\perp}, p_{\|}\right)$to $\left(\Gamma, \bar{p}_{\|}\right)$coordinates and applies the polyharmonic-spline algorithm described in appendix $\mathrm{C}$ to create an equally spaced and homogeneous grid in $\left(\Gamma, \bar{p}_{\|}\right)$coordinates. In this coordinate system, we perform the integration near poles and the analytic continuation in the same way as described in $\S \S 3.1$ and 3.2 , but using the relativistic parallel momentum associated with the pole from (3.15). For reasons of numerical performance, we use the integration based on (3.16) only if there is a pole within the integration domain. Otherwise, we employ the faster integration method based on (2.9) even in the relativistic case.

\section{Test cases and results}

In this section, we compare ALPS with known reference cases based on either our own or previously published results.

\subsection{Maxwellian distributions}

There are numerous codes for the hot-plasma dispersion relation in a plasma with Maxwellian or bi-Maxwellian background distributions. We use our code PLUME (Klein \& Howes 2015) for an electron-proton plasma and calculate the dispersion relations of Alfvén/ion-cyclotron (A/IC) and fast-magnetosonic/whistler (FM/W) waves. We then set up Maxwellian $f_{0}$ tables with the same parameters as those used with PLUME and calculate the dispersion relations based on these $f_{0}$ tables with ALPS. We compare the PLUME and ALPS results for quasi-parallel and quasi-perpendicular propagation in figure 1. The panels show both the real part of the frequency $\omega_{\mathrm{r}}$ and its imaginary part $\gamma$ as functions of the parallel and perpendicular wavenumbers, respectively. We use $\beta_{\perp j}=\beta_{\| j}=1$ for both protons and electrons, and $v_{\mathrm{A}} / c=10^{-4}$, where $\beta_{\perp j} \equiv 8 \pi n_{0 j} k_{\mathrm{B}} T_{\perp j} / B_{0}^{2}$ and $\beta_{\| j} \equiv 8 \pi n_{0 j} k_{\mathrm{B}} T_{\| j} / B_{0}^{2}$. We normalise all frequencies in units of the proton-cyclotron frequency $\Omega_{0 \mathrm{p}}$ and all length scales in units of the proton skin depth $d_{\mathrm{p}} \equiv v_{\mathrm{A}} / \Omega_{0 \mathrm{p}}$. The momentum-space resolution for the ALPS calculation in the quasi-parallel limit is $N_{\perp}=320, N_{\|}=640$, $P_{\max , \| \mathrm{p}}=8 m_{\mathrm{p}} v_{\mathrm{A}}$ and $P_{\max , \| \mathrm{e}}=0.19 m_{\mathrm{p}} v_{\mathrm{A}}$. In the quasi-perpendicular limit, we use $N_{\perp}=240, N_{\|}=480, P_{\max , \| \mathrm{p}}=6 m_{\mathrm{p}} v_{\mathrm{A}}$ and $P_{\max , \| \mathrm{e}}=0.14 m_{\mathrm{p}} v_{\mathrm{A}}$. In both cases, we set $P_{\max , \perp j}=P_{\max , \| j}, J_{\max }=10^{-45}, M_{\mathrm{I}}=5, M_{\mathrm{P}}=100, t_{\lim }=0.01$ and scan through $\boldsymbol{k}$-space in 256 logarithmically spaced steps from $k_{\|} d_{\mathrm{p}}=10^{-2}$ to $k_{\|} d_{\mathrm{p}}=10$ and $k_{\perp} d_{\mathrm{p}}=10^{-2}$ to $k_{\perp} d_{\mathrm{p}}=10$, respectively. We study the accuracy of the results depending on the resolution in $\S$ A.1. Figure 1 shows that ALPS reproduces these Maxwellian examples very well. We note that these plasma parameters represent typical solar-wind conditions at $1 \mathrm{au}$.

In order to illustrate another representation of the plasma dispersion relation, we show a comparison of dispersion maps from PLUME and ALPS in figure 2. Dispersion maps are diagrams of isocontours of constant $\lg |\operatorname{det} \mathcal{D}|$, where $\mathcal{D}$ is the tensor from (2.12), in the $\omega_{\mathrm{r}}-\gamma$ plane. Solutions to the hot-plasma dispersion relation appear as local minima in these diagrams. After calculating a dispersion map for a given $\boldsymbol{k}$, ALPS automatically determines the locations of its local minima in terms of $\omega_{\mathrm{r}}$ and $\gamma$. These minima represent ideal initial guesses for 

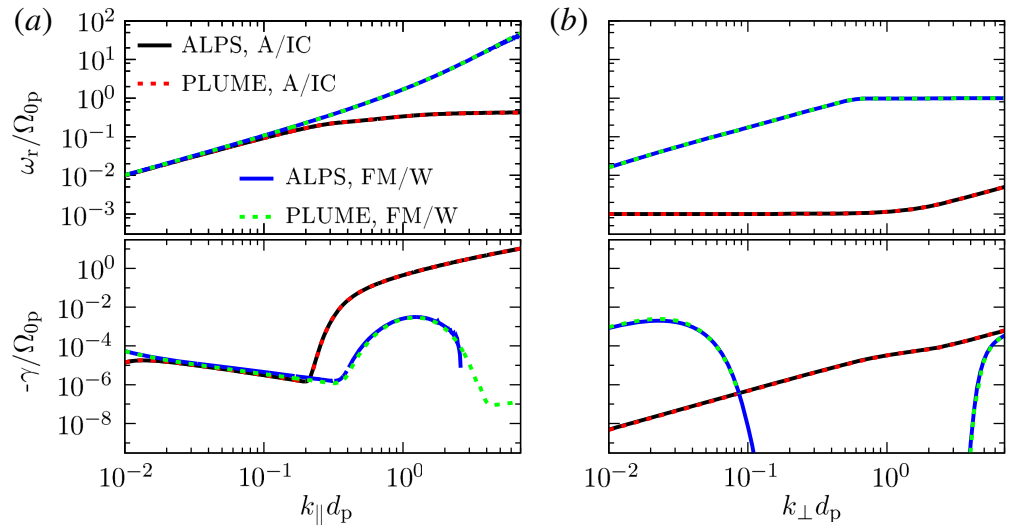

FIGURE 1. Dispersion relations for the A/IC wave and the FM/W wave in a Maxwellian plasma in quasi-parallel $(a)$ and quasi-perpendicular $(b)$ propagation. For the calculations shown on $(a)$, we keep $k_{\perp} d_{\mathrm{p}}=10^{-3}$ constant and scan through $k_{\|}$. For the calculations shown on $(b)$, we keep $k_{\|} d_{\mathrm{p}}=10^{-3}$ constant and scan through $k_{\perp}$. The A/IC mode in quasi-perpendicular propagation corresponds to the kinetic Alfvén wave (KAW) at $k_{\perp} d_{\mathrm{p}} \gtrsim$ $1 / \sqrt{\beta_{\| \mathrm{p}}}$. We compare ALPS with the standard Maxwellian solutions from PLUME for an electron-proton plasma with the same plasma parameters. Both numerical models agree well in both the real part $\omega_{\mathrm{r}}$ of the frequency and its imaginary part $\gamma$.

(a)

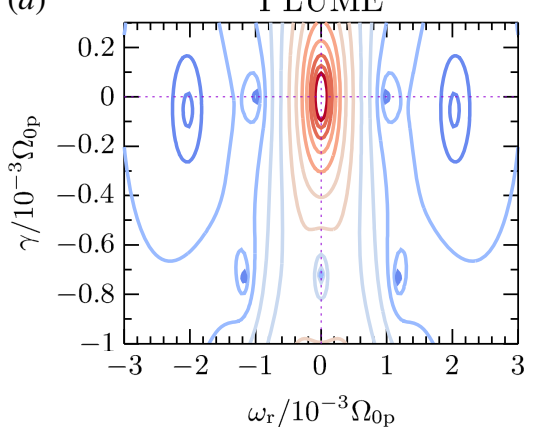

(b)

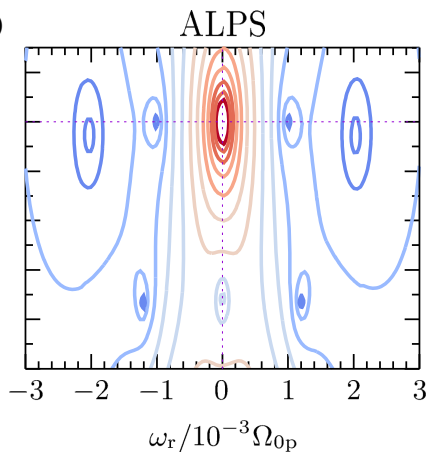

FIgURE 2. Comparison of dispersion maps from PLUME $(a)$ and ALPS $(b)$ for $k_{\perp} d_{\mathrm{p}}=$ $k_{\|} d_{\mathrm{p}}=10^{-3}$. The lines show isocontours of constant $\lg |\operatorname{det} \mathcal{D}|$. The colour scheme varies from small $\lg |\operatorname{det} \mathcal{D}|$ in blue to large $\lg |\operatorname{det} \mathcal{D}|$ in red. Minima in this map correspond to solutions to the hot-plasma dispersion relation.

the Newton-secant root-finding search when scanning through $\boldsymbol{k}$. Although the calculation of a dispersion map still requires the calculation of all $\chi_{j}$, it does not entail the application of the Newton-secant root-finding algorithm itself. We use a Maxwellian plasma model with $\beta_{\perp j}=\beta_{\| j}=1$ for both protons and electrons, $k_{\perp} d_{\mathrm{p}}=k_{\|} d_{\mathrm{p}}=10^{-3}$ and $v_{\mathrm{A}} / c=10^{-4}$. For the ALPS calculation, we use $N_{\perp}=240$, $N_{\|}=480, P_{\max , \| \mathrm{p}}=P_{\max , \perp \mathrm{p}}=6 m_{\mathrm{p}} v_{\mathrm{A}}, P_{\max , \| \mathrm{e}}=P_{\max , \perp \mathrm{e}}=0.14 m_{\mathrm{p}} v_{\mathrm{A}}, J_{\max }=10^{-45}$, $M_{\mathrm{I}}=5, M_{\mathrm{P}}=100$ and $t_{\mathrm{lim}}=0.01$. Both the PLUME and the ALPS calculations reveal seven solutions to the dispersion relation. We note that the point $\omega_{\mathrm{r}}=\gamma=0$ is a maximum and does not represent a solution to the dispersion relation. The 

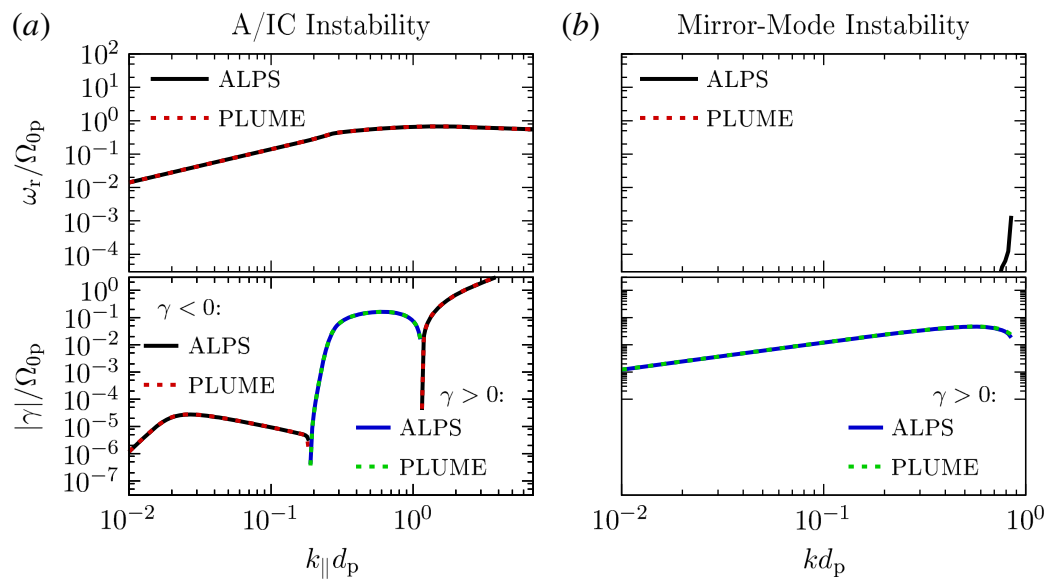

FIgURE 3. Comparison of dispersion relations for the A/IC instability $(a)$ and the mirrormode instability $(b)$ from PLUME and ALPS. We use $T_{\perp \mathrm{p}} / T_{\| \mathrm{p}}=3$. For the calculation of the A/IC instability, we keep $k_{\perp} d_{\mathrm{p}}=10^{-3}$ constant and scan through $k_{\|}$. For the calculation of the mirror-mode instability, we keep the angle $\theta=75^{\circ}$ constant and scan through $|\boldsymbol{k}|$.

solutions at $\omega_{\mathrm{r}}= \pm 10^{-3} \Omega_{0 \mathrm{p}}$ and $\gamma=-2.3 \times 10^{-10} \Omega_{0 \mathrm{p}}$ are the forward and backward propagating A/IC waves. The solutions at $\omega_{\mathrm{r}}= \pm 2 \times 10^{-3} \Omega_{0 \mathrm{p}}$ and $\gamma=-5.4 \times 10^{-5} \Omega_{0 \mathrm{p}}$ are the forward and backward propagating FM/W waves. The solutions at $\omega_{\mathrm{r}}= \pm 1.2 \times 10^{-3} \Omega_{0 \mathrm{p}}$ and $\gamma=-7.3 \times 10^{-4} \Omega_{0 \mathrm{p}}$ are the forward and backward propagating slow waves (ion-acoustic waves). Lastly, the solution at $\omega_{\mathrm{r}}=0$ and $\gamma=-7.2 \times 10^{-4} \Omega_{0 p}$ is the non-propagating slow mode, which is sometimes denoted 'entropy mode', (Verscharen et al. 2016; Verscharen, Chen \& Wicks 2017). The comparison of both panels in figure 2 shows that ALPS reproduces these seven plasma modes under typical solar-wind conditions in the Maxwellian limit.

\subsection{Anisotropic bi-Maxwellian distributions}

PLUME, like most other standard hot-plasma dispersion-relation solvers, allows us to use anisotropic bi-Maxwellian representations for the background distribution functions. Such a configuration can lead to instability if the temperature anisotropy exceeds the threshold for an anisotropy-driven plasma instability. As an example for a propagating instability, we calculate the dispersion relation for the parallel A/IC instability (Harris 1961; Davidson \& Ogden 1975; Yoon et al. 2010), and as an example for a non-propagating instability, we calculate the dispersion relation for the mirror-mode instability (Rudakov \& Sagdeev 1961; Tajiri 1967; Southwood \& Kivelson 1993). The thresholds for both of these instabilities fulfil $T_{\perp \mathrm{p}}>T_{\| \mathrm{p}}$. For this demonstration, we use PLUME to calculate $\omega_{\mathrm{r}}$ and $\gamma$ as functions of the wavenumber in a plasma with bi-Maxwellian protons and Maxwellian electrons using $\beta_{\| \mathrm{p}}=\beta_{\| \mathrm{e}}=\beta_{\perp \mathrm{e}}=1, T_{\perp \mathrm{p}} / T_{\| \mathrm{p}}=3$ and $v_{\mathrm{A}} / c=10^{-4}$. We then set-up bi-Maxwellian $f_{0}$ tables with the same parameters and calculate the dispersion relations for both instabilities with ALPS. We show the results in figure 3. For the ALPS calculation, we use $N_{\perp}=320, N_{\|}=640, P_{\max , \| \mathrm{p}}=8 m_{\mathrm{p}} v_{\mathrm{A}}, P_{\max , \perp \mathrm{p}}=13.9 m_{\mathrm{p}} v_{\mathrm{A}}$, $P_{\max , \| \mathrm{e}}=P_{\max , \perp \mathrm{e}}=0.19 m_{\mathrm{p}} v_{\mathrm{A}}, J_{\max }=10^{-45}, M_{\mathrm{I}}=5, M_{\mathrm{P}}=100$ and $t_{\mathrm{lim}}=0.01$. For the $\mathrm{A} / \mathrm{IC}$ instability, we scan through $k_{\|}$from $k_{\|} d_{\mathrm{p}}=10^{-2}$ to $k_{\|} d_{\mathrm{p}}=10$ in 256 


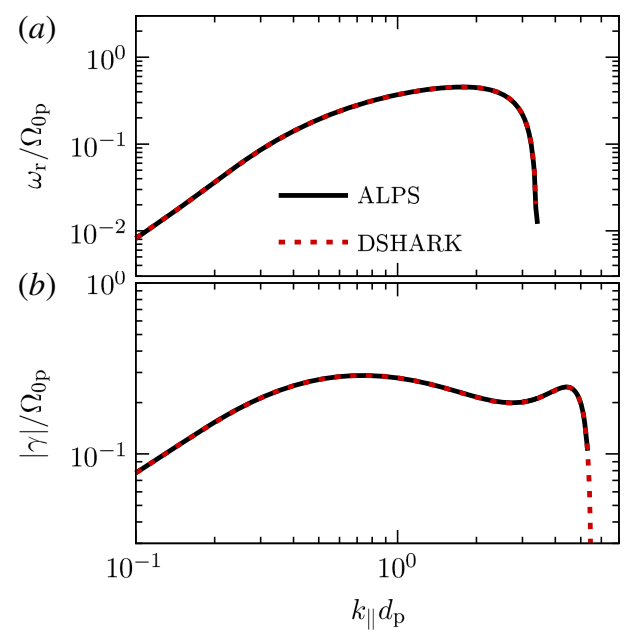

FIGURE 4. Comparison of dispersion relations of the FM/W instability in a $\kappa$-distributed plasma from DSHARK and ALPS. In both plasma models, we keep $\theta=0.001^{\circ}$ constant and scan through $k_{\|}$. (a) shows the real part of the wave frequency, and $(b)$ shows its imaginary part.

logarithmically spaced steps. For the mirror-mode instability, we scan through $k$ from $k d_{\mathrm{p}}=10^{-2}$ to $k d_{\mathrm{p}}=5$ in 154 logarithmically spaced steps and keep the angle $\theta \equiv \arcsin \left(k_{\perp} / k\right)=75^{\circ}$ constant. We study the accuracy of these results depending on the resolution in $\S \mathrm{A} .2$.

Both PLUME and ALPS show that the A/IC wave and the mirror mode are unstable in different wave-vector ranges for the given parameter set. The good agreement between the PLUME solutions and the ALPS solutions shows that ALPS successfully calculates the dispersion relations of both instabilities in a bi-Maxwellian plasma. Although the correct mirror mode exhibits $\omega_{\mathrm{r}}=0$, the ALPS solution shows a very small yet finite $\omega_{\mathrm{r}}$. We discuss this error and its dependence on the velocity-space resolution in $\S \mathrm{A} .2$.

\subsection{Anisotropic $\kappa$-distributions}

Astfalk et al. (2015) developed the code DSHARK to calculate dispersion relations in plasmas with bi- $\kappa$-distributions. As one example, these authors discuss the FM/W instability in an anisotropic electron-proton plasma with $\kappa_{\mathrm{p}}=\kappa_{\mathrm{e}}=8, \beta_{\| \mathrm{p}}=2, \beta_{\| \mathrm{e}}=$ 4, $T_{\perp \mathrm{p}} / T_{\| \mathrm{p}}=0.4$ and $T_{\perp \mathrm{e}} / T_{\| \mathrm{e}}=0.5$ (see figure 1 from Astfalk et al. 2015). The angle between $\boldsymbol{k}$ and $\boldsymbol{B}_{0}$ is constant for this calculation and set to $\theta=0.001^{\circ}$. We use DSHARK to reproduce this test case and set up $\kappa$-distributed $f_{0}$ tables with the same parameters in order to compare the DSHARK results with ALPS. We show this comparison in figure 4. In ALPS, we use $N_{\perp}=400, N_{\|}=800, P_{\max , \| \mathrm{p}}=10 m_{\mathrm{p}} v_{\mathrm{A}}$, $P_{\text {max }, \perp \mathrm{p}}=6.32 m_{\mathrm{p}} v_{\mathrm{A}}, P_{\max , \| \mathrm{e}}=0.33 m_{\mathrm{p}} v_{\mathrm{A}}, P_{\max , \perp \mathrm{e}}=0.23 m_{\mathrm{p}} v_{\mathrm{A}}, J_{\max }=10^{-45}, M_{\mathrm{I}}=5$, $M_{\mathrm{P}}=500, t_{\mathrm{lim}}=0.01$ and $v_{\mathrm{A}} / c=10^{-4}$. We scan through $k_{\|}$from $k_{\|} d_{p}=10^{-1}$ to $k_{\|} d_{\mathrm{p}} \approx 3.42$, where $\omega_{\mathrm{r}}$ changes its sign after 87 logarithmically spaced steps.

ALPS reproduces the DSHARK results for the FM/W instability well. The results also agree with the previous work by Lazar et al. (2011). 

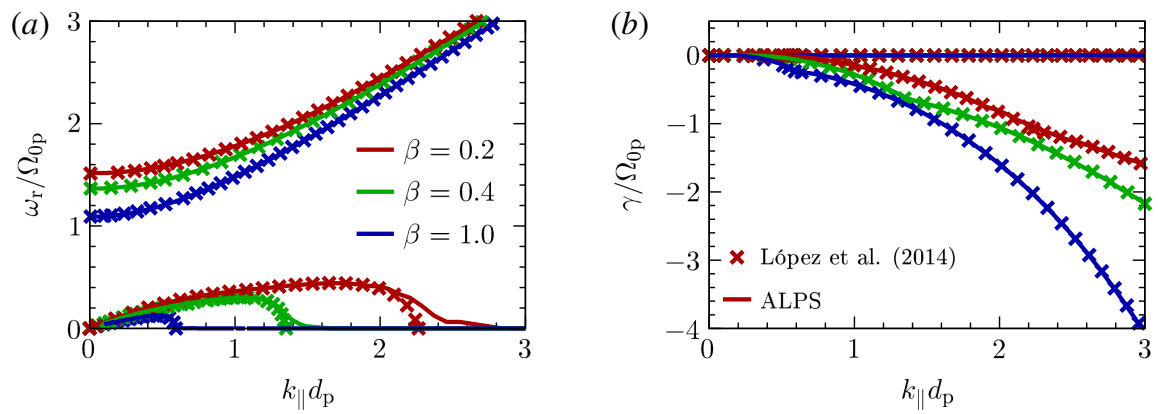

FIGURE 5. Dispersion relations of the quasi-parallel A/IC wave (solutions at low $\omega_{\mathrm{r}}$ ) and the ordinary wave (solutions at high $\omega_{\mathrm{r}}$ ) in a relativistic electron-positron pair plasma with Jüttner distributions. We keep $k_{\perp} d_{\mathrm{p}}=10^{-3}$ constant and scan through $k_{\|}$. (a) shows the real part of the frequency, and $(b)$ shows the imaginary part of the frequency. The lines show ALPS solutions, and the crosses show the results from figure 1 of López et al. (2014). The three colours correspond to $\beta_{\| \mathrm{p}}=\beta_{\| \mathrm{e}}=0.2$ (red), $\beta_{\| \mathrm{p}}=\beta_{\| \mathrm{e}}=0.4$ (green) and $\beta_{\| \mathrm{p}}=\beta_{\| \mathrm{e}}=1.0$ (blue) in both panels and for both modes.

\subsection{Relativistic Jüttner distributions}

As one example for a dispersion relation in a relativistic plasma, we reproduce the results by López et al. (2014) for an electron-positron pair plasma with a Jüttner distribution using $v_{\mathrm{A}} / c=1, m_{\mathrm{p}}=m_{\mathrm{e}}, \beta_{\| \mathrm{p}}=\beta_{\| \mathrm{e}}=(0.2,0.4,1.0)$ and $T_{\perp j}=T_{\| j}$ for both positrons and electrons. We set up a Jüttner-distributed $f_{0}$ table with the same parameters and calculate the dispersion relations of the $\mathrm{A} / \mathrm{IC}$ wave and the Ordinary wave (O-mode) in the plasma, keeping the perpendicular wavenumber constant at $k_{\perp} d_{\mathrm{p}}=10^{-3}$. We use $N_{\perp}=30, N_{\|}=60, P_{\max }=5 m_{\mathrm{p}} v_{\mathrm{A}}, J_{\max }=10^{-45}, M_{\mathrm{I}}=5, M_{\mathrm{P}}=300$ and $t_{\text {lim }}=0.01$. Our interpolation method transforms the $\left(p_{\perp}, p_{\|}\right)$grid to the $\left(\Gamma, \bar{p}_{\|}\right)$ grid with $N_{\Gamma}=500$ and $N_{\bar{p}_{\|}}=500$ steps in $\Gamma$ and $\bar{p}_{\|}$, respectively. We scan through $k_{\|}$from $k_{\|} d_{\mathrm{p}}=10^{-1}$ to $k_{\|} d_{\mathrm{p}}=10$ in 128 logarithmically spaced steps. We show the results in figure 5. López et al. (2014) show their results for these parameters in their figure 1. Our comparison with the ALPS dispersion relation in figure 5 shows a good agreement and confirms our relativistic model. The deviation between the results from López et al. (2014) and ALPS is only visible in the real part of the frequency at the large- $k_{\|} /$low- $\omega_{\mathrm{r}}$ end of the $\mathrm{A} / \mathrm{IC}$ branches.

\section{Discussion and conclusions}

ALPS solves the relativistic and non-relativistic hot-plasma dispersion relations in a plasma with arbitrary background distribution functions. We have benchmarked ALPS against existing codes by comparing dispersion relations for waves and instabilities in Maxwellian, bi-Maxwellian, $\kappa$-distributed and relativistic Jüttner-distributed plasmas. In all cases, we find that ALPS agrees well with existing codes. This finding encourages us to apply ALPS to yet unexplored plasma environments in future work. We note that we have only tested ALPS in the regime $k \rho_{\mathrm{e}} \ll 1$ and $k d_{\mathrm{e}} \ll 1$ thus far, where $\rho_{\mathrm{e}} \equiv w_{\perp \mathrm{e}} /\left|\Omega_{0 \mathrm{e}}\right|$. There is no numerical limitation, however, that prevents us from applying the code to larger wavenumbers at or beyond electron scales.

An important application of ALPS will be the analysis of distribution functions measured by spacecraft in the solar wind. ALPS includes the necessary numerical framework to preprocess and format the spacecraft data so that they can serve as 
$f_{0}$ tables for direct input (see appendix C). Especially, the upcoming missions Solar Orbiter and Parker Solar Probe will deliver plasma measurements with unprecedented energy and time resolution in the solar wind that will serve as the ideal input for ALPS. The vast majority of previous kinetic studies of waves and instabilities relied on bi-Maxwellian fits to the observed distribution functions and the use of a standard bi-Maxwellian code to solve the hot-plasma dispersion relation such as WHAMP, PLUME or NHDS. Our approach allows us, however, to relax the bi-Maxwellian assumption and to analyse the plasma behaviour more realistically. A great advantage of standard bi-Maxwellian solvers is their very high speed when solving the dispersion relation. The plots of dispersion relations shown in this paper require an ALPS run time between about one and ten hours on 32 processor cores, while PLUME solves the same dispersion relation for a bi-Maxwellian plasma within a few seconds. Therefore, we recommend the use of standard bi-Maxwellian solvers or solvers for $\kappa$-distributed plasmas to determine an adequate initial guess before using ALPS whenever the $f_{0}$ table is close to a bi-Maxwellian or $\kappa$-distribution, respectively. Future comparisons of the results from standard codes such as PLUME with the results from ALPS will help to evaluate the quality of the previous bi-Maxwellian approaches and to refine our understanding of the role of instabilities in collisionless plasmas based on the actual distribution functions. For instance, our knowledge of the realistic value of certain instability thresholds is still very limited. Some in situ observations of kinetic plasma features in the solar wind lie above the thresholds of kinetic instabilities when calculated based on bi-Maxwellian background distributions (see, for example, Isenberg 2012). The general conjecture is, however, that the plasma is limited by the lowest instability threshold. A more realistic calculation based on the actual distribution functions may resolve this discrepancy. This concept applies, for example, to anisotropy-driven instabilities such as the A/IC instability (Hellinger et al. 2006; Bale et al. 2009; Maruca, Kasper \& Gary 2012) or beam-driven instabilities such as the FM/W instability (Reisenfeld et al. 2001; Verscharen, Bourouaine \& Chandran 2013; Verscharen \& Chandran 2013). Also non-thermal electron configurations, which are known to carry a significant heat flux into the solar wind, require a non-bi-Maxwellian representation for the determination of the relevant instabilities that limit their heat flux (Feldman et al. 1975; Pilipp et al. 1987a; Pulupa et al. 2011; Salem et al. 2013). Another field of application of ALPS is the study of highly non-thermal plasma configurations related to reconnection events (Phan et al. 2006; Gosling 2007; Gosling et al. 2007; Egedal, Daughton \& Le 2012; Egedal, Le \& Daughton 2013). We also emphasise the applicability of ALPS for the determination of dispersion relations using distributions from numerical plasma simulations. Particle-in-cell or Eulerian plasma codes generate data directly suitable as $f_{0}$ tables for ALPS. Some of these numerical simulations use (realistically or artificially) relativistic plasma conditions. Therefore, ALPS's ability to include relativistic effects will be very useful for the study of the wave properties and the stability of simulated plasmas.

Our resolution studies in appendix A offer some insight into the necessary resolution of the $f_{0}$ tables for a reliable determination of the plasma dispersion relation. In the shown applications, a minimum resolution of about $N_{\perp}=40$ and $N_{\|}=80$ has proven to be necessary for a good agreement between ALPS and the test results for (bi-)Maxwellian distributions. In a future extension of ALPS, we will include Nyquist's method to automatically determine the stability of directly observed distribution functions (Klein et al. 2017, 2018). We also note that, in cases with $k_{\|} \ll k_{\perp}$ and $\omega_{\mathrm{r}} \ll \Omega_{\mathrm{p} 0}$, the gyrokinetic limit is an appropriate simplification to the 
full hot-plasma dispersion relation (Catto 1978; Howes et al. 2006; Kunz et al. 2015; Camporeale \& Burgess 2017). The gyrokinetic dispersion relation does not require the sum over all $n \neq 0$ as in (2.9). Considering these lower numerical demands, it would be worthwhile to develop a gyrokinetic version of ALPS in the future.

\section{Acknowledgement}

We appreciate helpful comments and contributions from S. Markovskii and T. Brackett. The ALPS collaboration appreciates support from NASA grant NNX16AG81G. We present more details about the numerics on the website http://www.alps.space. The ALPS source code will be made publicly available on this website after our initial science phase. Computations were performed on Trillian, a Cray XE6m-200 supercomputer at UNH supported by the NSF MRI programme under grant PHY-1229408. D.V. was supported by the STFC Ernest Rutherford Fellowship ST/P003826/1. B.D.G.C. was supported in part by NASA grants NNX15AI80G and NNX17AI18G and NSF grant PHY-1500041. Work at UC Berkeley was supported in part by NSF/SHINE grant AGS-1622498 and NASA grants NNX14AJ70G, NNX16AI59G and NNX16AP95G.

\section{Appendix A. Resolution studies}

In order to understand the required resolution of the $f_{0}$ tables for calculations with ALPS, we compare results from PLUME with results from ALPS for the same plasma parameters using different resolutions in this appendix. For all calculations, we use $\beta_{\| \mathrm{p}}=\beta_{\| \mathrm{e}}=1, T_{\perp \mathrm{e}} / T_{\| \mathrm{e}}=1, J_{\max }=10^{-45}, M_{\mathrm{I}}=5, M_{\mathrm{P}}=100$ and $t_{\text {lim }}=0.01$. A careful re-analysis of the A/IC-wave solutions using variations in $M_{\mathrm{I}}$ and $M_{\mathrm{P}}$ over one order of magnitude each (not shown) reveals that the accuracy of the solutions depends only moderately on the numerical values of these parameters. We use $P_{\max , \| \mathrm{p}}$ as a free parameter and set $N_{\|}=2 N_{\perp}, P_{\max , \| \mathrm{e}}=P_{\max , \perp \mathrm{e}}=P_{\max , \| \mathrm{p}} \sqrt{m_{\mathrm{e}} / m_{\mathrm{p}}}$ and $P_{\max , \perp \mathrm{p}}=P_{\max , \| \mathrm{p}} \sqrt{T_{\perp \mathrm{p}} / T_{\| \mathrm{p}}}$. We apply the same resolutions for the scans in $\boldsymbol{k}$-space as those used to derive the corresponding figures in $\S 4$. We define the resolution in momentum as $\Delta w_{j} \equiv P_{\max , \| j} /\left(N_{\|} m_{j} w_{\| j}\right)$ and the accuracy of the solution as $\Delta \omega_{\mathrm{r}} / \omega_{\mathrm{r}} \equiv\left|\omega_{\mathrm{r}, \mathrm{ALPS}}-\omega_{\mathrm{r}, \text { PLUME }}\right| / \omega_{\mathrm{r}, \text { PLUME }}$, where $\omega_{\mathrm{r}, \text { ALPS }}$ is the solution from ALPS and $\omega_{\mathrm{r}, \mathrm{PLUME}}$ is the solution from PLUME. For $\kappa$-distributions, the appropriate resolution depends on both $\beta_{\| j}$ and $\kappa$. Instead of giving general guidelines for the resolution, we, therefore, recommend case-by-case convergence studies when calculating dispersion relations in plasmas with $\kappa$-distributions.

\section{A.1. Maxwellian distributions}

In figure 6, we show a resolution study for the A/IC wave in quasi-parallel propagation in an isotropic Maxwellian plasma. This figure complements our solutions shown in figure 1 . The four panels represent different values of $P_{\max , \| j}$. In each panel, the diagram at the top compares the real part of the frequency from five ALPS calculations with different $\Delta w_{j}$ to the Maxwellian solutions from PLUME. The diagram at the bottom compares the ratio between $\omega_{\mathrm{r}}$ from the five ALPS calculations and $\omega_{\mathrm{r}}$ from PLUME. The line associated with a given parameter run ends whenever ALPS loses the solution as it scans through $k_{\|}$. For the parameters used in figure 6, $P_{\text {max, } \| p}=8 m_{\mathrm{p}} w_{\mathrm{p}}$ with a resolution finer than $\Delta w_{j}=0.1$ leads to a very good agreement with the PLUME solutions for $\omega_{\mathrm{r}}$. For wavenumbers below $1 / d_{\mathrm{p}}$, a lower value of $P_{\max , \| \mathrm{p}}$ is sufficient. Figure 7 shows the same as figure 6, but giving the imaginary part 

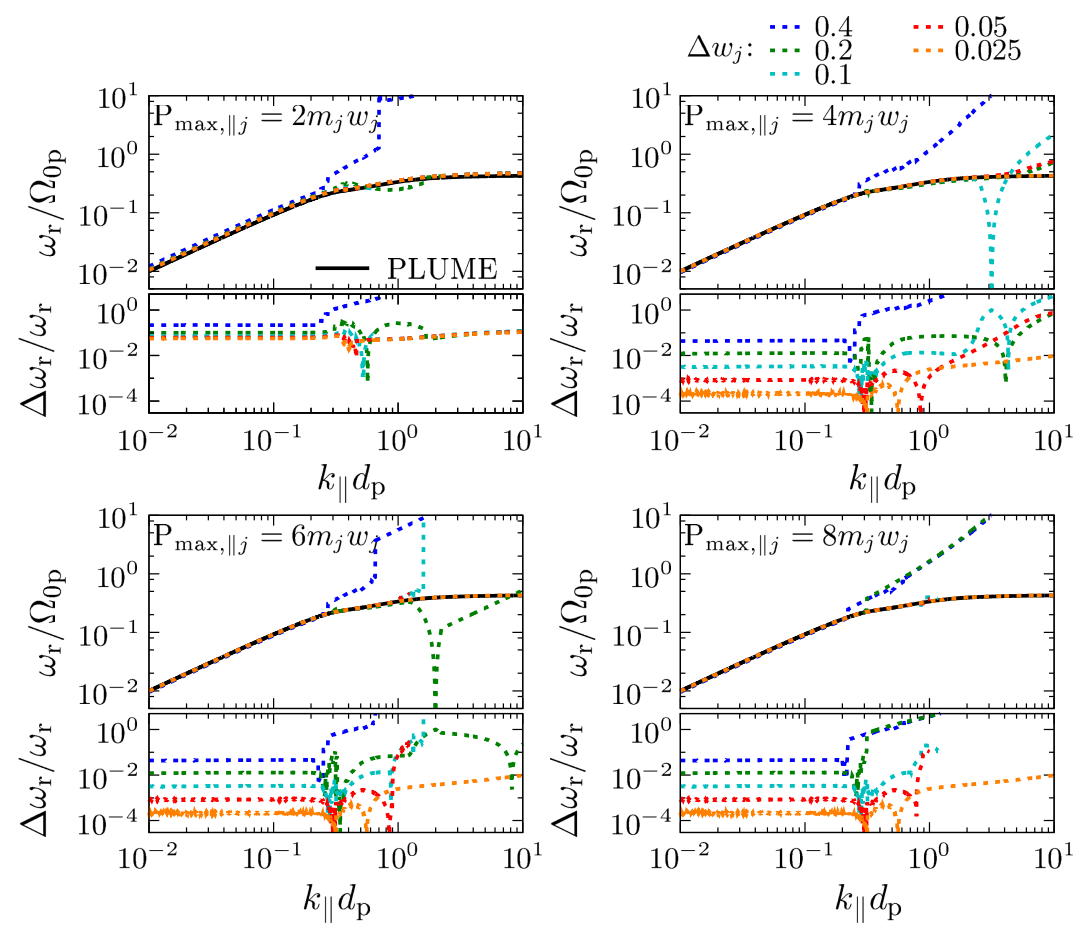

FIGURE 6. Resolution study for the real part of the frequency for the A/IC-wave solution in quasi-parallel propagation. We keep $k_{\perp} d_{\mathrm{p}}=10^{-3}$ constant and scan through $k_{\|}$.

of the frequency instead of its real part. This figure confirms our finding regarding the optimal resolution.

Figures 8 and 9 show the same as figures 6 and 7, but for quasi-perpendicular propagation instead of quasi-parallel propagation. The required resolution is lower in the quasi-perpendicular case than in the quasi-parallel case. The solutions with $P_{\max , \| \mathrm{p}}=4 m_{\mathrm{p}} w_{\mathrm{p}}$ and $\Delta w_{j} \leqslant 0.1$ lead to a very good agreement between the PLUME and ALPS solutions.

\section{A.2. Anisotropic bi-Maxwellian distributions}

In addition to our Maxwellian test, we study the dependence of the ALPS solutions on the resolutions for the bi-Maxwellian case with $T_{\perp \mathrm{p}} / T_{\| \mathrm{p}}=3$ as shown in figure 3. Figure 10 compares ALPS solutions for the A/IC instability in quasi-parallel propagation for different values of $P_{\max , \| \mathrm{p}}$ and $\Delta w_{j}$ with the solutions from PLUME for the real part of the frequency. Figure 11 compares PLUME and ALPS solutions for the imaginary part of the frequency. The solutions with $P_{\max , \| \mathrm{p}}=4 m_{\mathrm{p}} w_{\| \mathrm{p}}$ and $\Delta w_{j} \leqslant 0.05$ lead to a good agreement between PLUME and ALPS in both $\omega_{\mathrm{r}}$ and $\gamma$.

In figure 12, we study the dependence of the solutions on the resolution for the mirror-mode instability with the same parameters as in figure 3 . The correct solution of the mirror-mode instability has $\omega_{\mathrm{r}}=0$; however, the ALPS solutions have finite values $\omega_{\mathrm{r}} \neq 0$. The value of $\omega_{\mathrm{r}}$ decreases with increasing $\Delta w_{j}$. As Southwood \& Kivelson (1993) point out, the mirror-mode instability is strongly influenced by particles with $p_{\|} \approx 0$. The error in frequency $\Delta \omega_{\mathrm{r}}$ is determined by the resolution of the momentum grid around $p_{\|}=0$, where $\Delta \omega_{\mathrm{r}} \sim k_{\|} w_{j} \Delta w_{j}$. Figure 13 shows the 

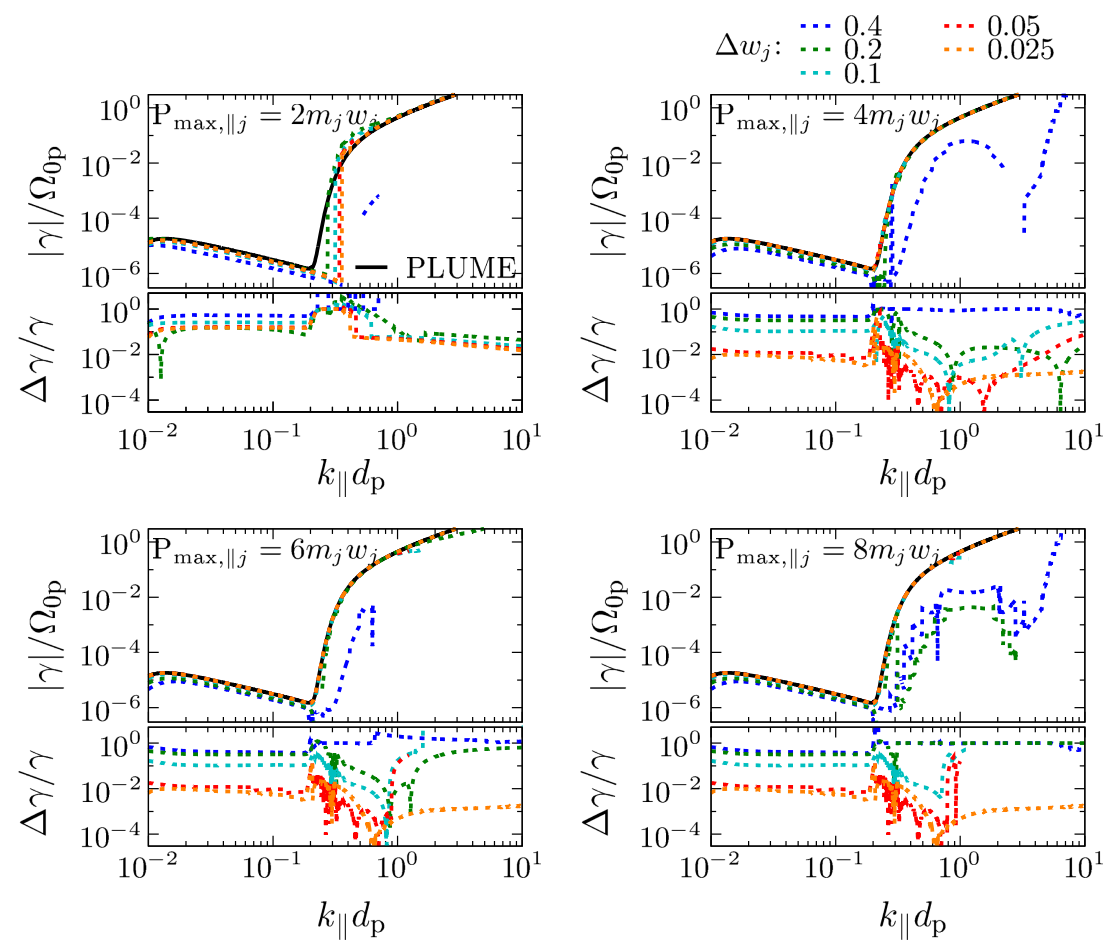

FIGURE 7. Resolution study for the imaginary part of the frequency for the A/IC-wave solution in quasi-parallel propagation. We keep $k_{\perp} d_{\mathrm{p}}=10^{-3}$ constant and scan through $k_{\|}$.

comparison of the imaginary part of the mirror-mode solutions. Like in the case of the A/IC instability, a resolution with $P_{\max , \| p}=4 m_{\mathrm{p}} w_{\| \mathrm{p}}$ and $\Delta w_{j} \leqslant 0.05$ leads to a good agreement between PLUME and ALPS. We note that there is a non-monotonic behaviour in the accuracy of the solutions in some cases (see, for example, the solutions for $\Delta w_{j}=0.2, \Delta w_{j}=0.1$, and $\Delta w_{j}=0.05$ in figure 13). This illustrates that an increase in resolution sometimes causes the code to lose a solution that it successfully finds at lower resolutions. Since this numerical problem eventually resolves itself after increasing the resolution further, we recommend applying a higher resolution whenever the code loses a solution.

\section{Appendix B. Levenberg-Marquardt fit}

For the hybrid analytic continuation, ALPS fits the $f_{0}$ table with a combination of pre-described algebraic expressions as described in \$3.2. We employ a LevenbergMarquardt algorithm (Levenberg 1944; Marquardt 1963) to fit the distribution functions in $p_{\|}$with a superposition of an arbitrary number of Maxwellian distributions, $\kappa$-distributions and Jüttner distributions. The user can freely choose the number of fits and their superposition. We evaluate different fit parameters for each given value of $p_{\perp}$. We define the Maxwellian fitting function as

$$
F_{\mathrm{M}}\left(\hat{p}_{\|}\right)=u_{1} \exp \left[-y \hat{p}_{\perp}^{2}-u_{2}\left(\hat{p}_{\|}-u_{3}\right)^{2}\right]
$$

where $u_{k}$ are the fit parameters, $y$ is a constant user-defined parameter and $\hat{p}_{\perp}$ and $\hat{p}_{\|}$ are the normalised perpendicular and parallel momenta. The parameter $y$ compensates 

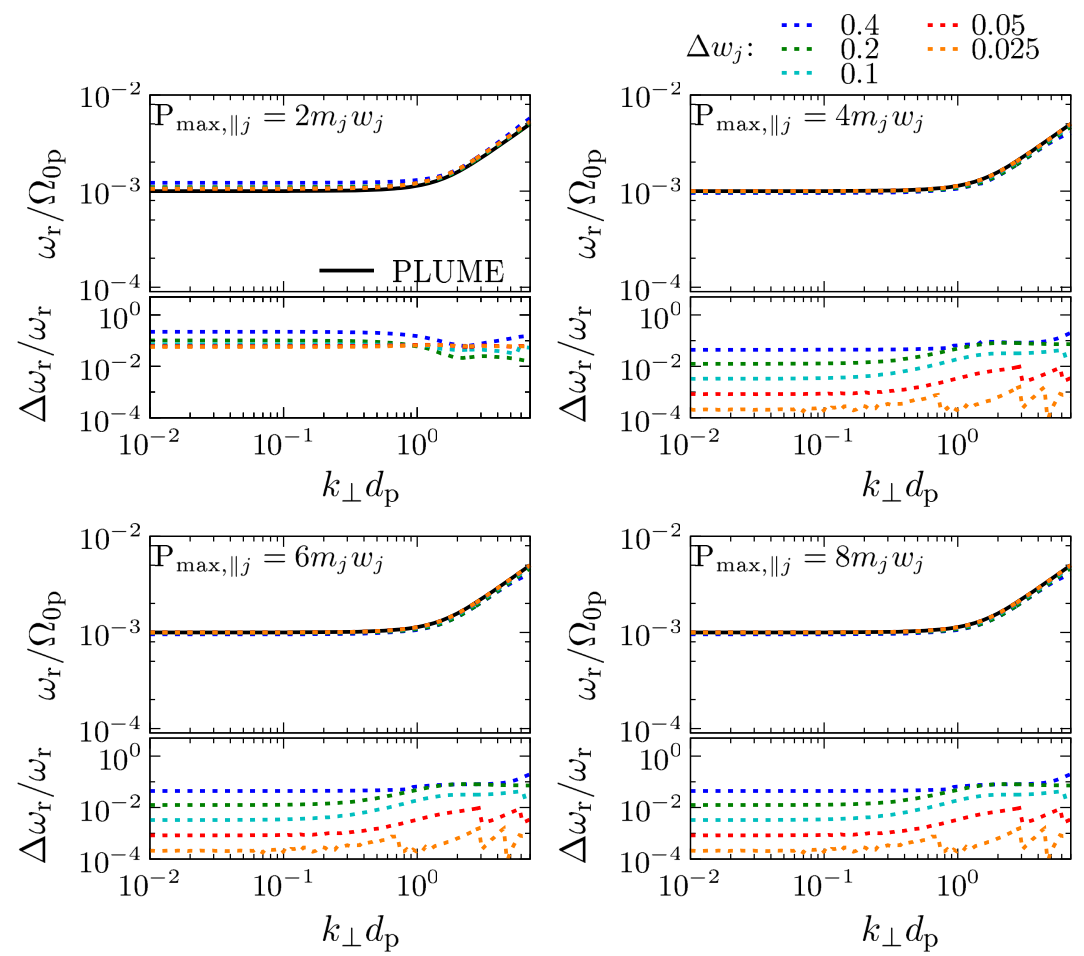

FIGURE 8. Resolution study for the real part of the frequency for the A/IC-wave solution in quasi-perpendicular propagation. We keep $k_{\|} d_{\mathrm{p}}=10^{-3}$ constant and scan through $k_{\perp}$.

the otherwise strong $p_{\perp}$-dependence of $u_{1}$, making the fit more reliable. It is constant for all $p_{\perp}$. We choose this expression rather than a fit in $p_{\perp}$ since it provides a greater flexibility in the $p_{\perp}$-domain compared to a two-dimensional fit in $p_{\perp}$ and $p_{\|}$. The best choice for $y$ is $\beta_{\perp j} m_{\mathrm{p}} / m_{j}$. The standard normalisation in ALPS uses $\hat{p}_{\perp}=p_{\perp} / m_{\mathrm{p}} v_{\mathrm{A}}$ and $\hat{p}_{\|}=p_{\|} / m_{\mathrm{p}} v_{\mathrm{A}}$. In cases with $\kappa$-distributed plasma components, we use

$$
F_{\kappa}\left(\hat{p}_{\|}\right)=u_{1}\left[1+u_{2}\left(\hat{p}_{\|}-u_{3}\right)^{2}+y \hat{p}_{\perp}^{2}\right]^{u_{4}} .
$$

In cases with Jüttner-distributed plasma components, we use

$$
F_{\mathrm{J}}=u_{1} \exp (-y \Gamma)
$$

In this case, the best choice for $y$ is $2 c^{2} / w_{j}^{2}$. These fitting relations are easily extendable by the user to cover more general functions as needed.

We denote the discretised $f_{0}$ table of species $j$ at constant $p_{\perp}$ as $\hat{f}_{i, j}\left(\hat{p}_{\|, i}\right)$, the discrete steps in $\hat{p}_{\|}$as $\hat{p}_{\|, i}$, the vector of all fit parameters as $\boldsymbol{u}$ and the sum of all fit functions as $F\left(\hat{p}_{\|}\right)$. In the Jüttner-distributed cases, the coordinates are replaced with $\Gamma$ and $\bar{p}_{\|}$accordingly. We define the residuals as $s_{i} \equiv \hat{f}_{i, j}-F\left(\hat{p}_{\|, i}\right)$ and define $C \equiv \sum_{i} s_{i}^{2}$. We denote the Jacobian of $\hat{\boldsymbol{f}}_{j}$ with respect to $\boldsymbol{u}$ as $\boldsymbol{J}$. We use a superposition of analytical expressions for the Jacobian based on the given form of $F\left(\hat{p}_{\|}\right)$. The Levenberg-Marquardt algorithm uses an iterative step to update $\boldsymbol{u}$ of the form

$$
\boldsymbol{u}_{\text {new }}=\boldsymbol{u}+\left[\boldsymbol{J}^{\top} \boldsymbol{J}+\lambda \operatorname{diag}\left(\boldsymbol{J}^{\top} \boldsymbol{J}\right)\right]^{-1} \boldsymbol{J}^{\top} \boldsymbol{s},
$$



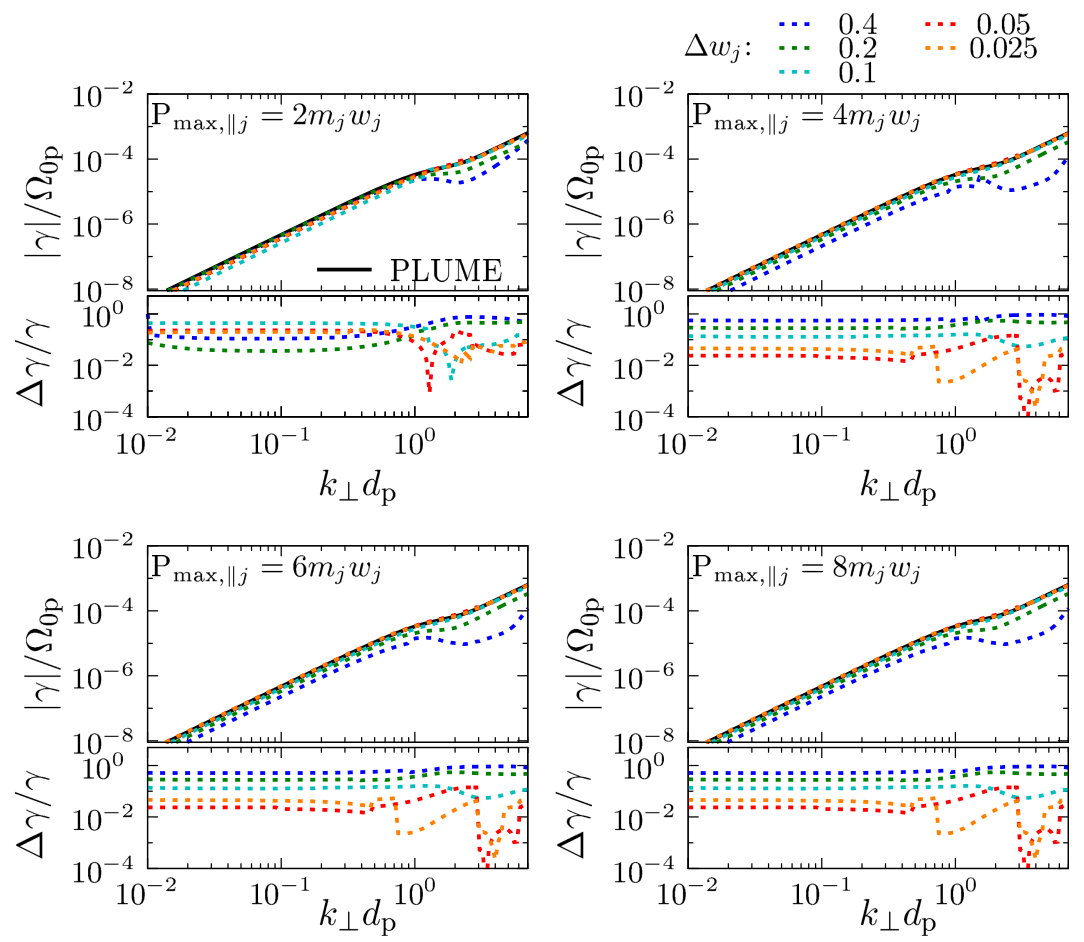

FIGURE 9. Resolution study for the imaginary part of the frequency for the A/IC-wave solution in quasi-perpendicular propagation. We keep $k_{\|} d_{\mathrm{p}}=10^{-3}$ constant and scan through $k_{\perp}$.

where $\lambda$ is a user-defined scalar. For the matrix inversion in (B 4), we use the $\boldsymbol{L} \boldsymbol{U}$-factorisation. Then we calculate the residuals $\boldsymbol{s}_{\text {new }}$ based on $\boldsymbol{u}_{\text {new }}$ and determine $C_{\text {new }}=\left|\boldsymbol{s}_{\text {new }}\right|^{2}$. If $C_{\text {new }} \leqslant C$, we set $\boldsymbol{u}$ to $\boldsymbol{u}_{\text {new }}$, reduce $\lambda$ by a constant factor $\lambda_{\mathrm{f}}$ (user-defined, standard value is 10) and repeat the procedure. If $C_{\text {new }}>C$, we discard $u_{\text {new }}$, increase $\lambda$ by the constant factor $\lambda_{\mathrm{f}}$ and repeat the procedure. In this way, we iteratively determine the fit parameters $\boldsymbol{u}$ until the fit converges (i.e. $C \leqslant \epsilon$ with a user-defined $\epsilon$ ), or until the number of iterations reaches a user-defined maximum value. ALPS writes the fitted distribution into a separate output file so that a direct comparison with the original input distribution is possible.

\section{Appendix C. The smoothed thin-plate spline interpolation}

Spacecraft or other plasma data are typically not available on a dense Cartesian grid like the grid required for an $f_{0}$ table in ALPS. Therefore, our code includes an interpolation algorithm that fills gaps between data points. ALPS uses the same interpolation algorithm to create an equidistant grid in $\left(\Gamma, \bar{p}_{\|}\right)$space after the coordinate transformation in cases with relativistic poles. We use a polyharmonicspline interpolation with the radial basis function of a thin-plate spline with smoothing (Powell 1994; Donato \& Belongie 2002). For each species, we begin with the 'coarse' distribution function $\hat{f}_{\mathrm{c}, \mu}$ which is given by $N_{\mathrm{c}}$ data points (index $\mu=1 \ldots N_{\mathrm{c}}$ ) with the associated coarse momentum coordinates $\hat{p}_{\perp \mathrm{c}, \mu}$ and $\hat{p}_{\| \mathrm{c}, \mu}$. The set $\left(\hat{f}_{\mathrm{c}, \mu}, \hat{p}_{\perp \mathrm{c}, \mu}, \hat{p}_{\| \mathrm{c}, \mu}\right)$ forms one data point. The coarse grid is typically not equally distributed in momentum space. 

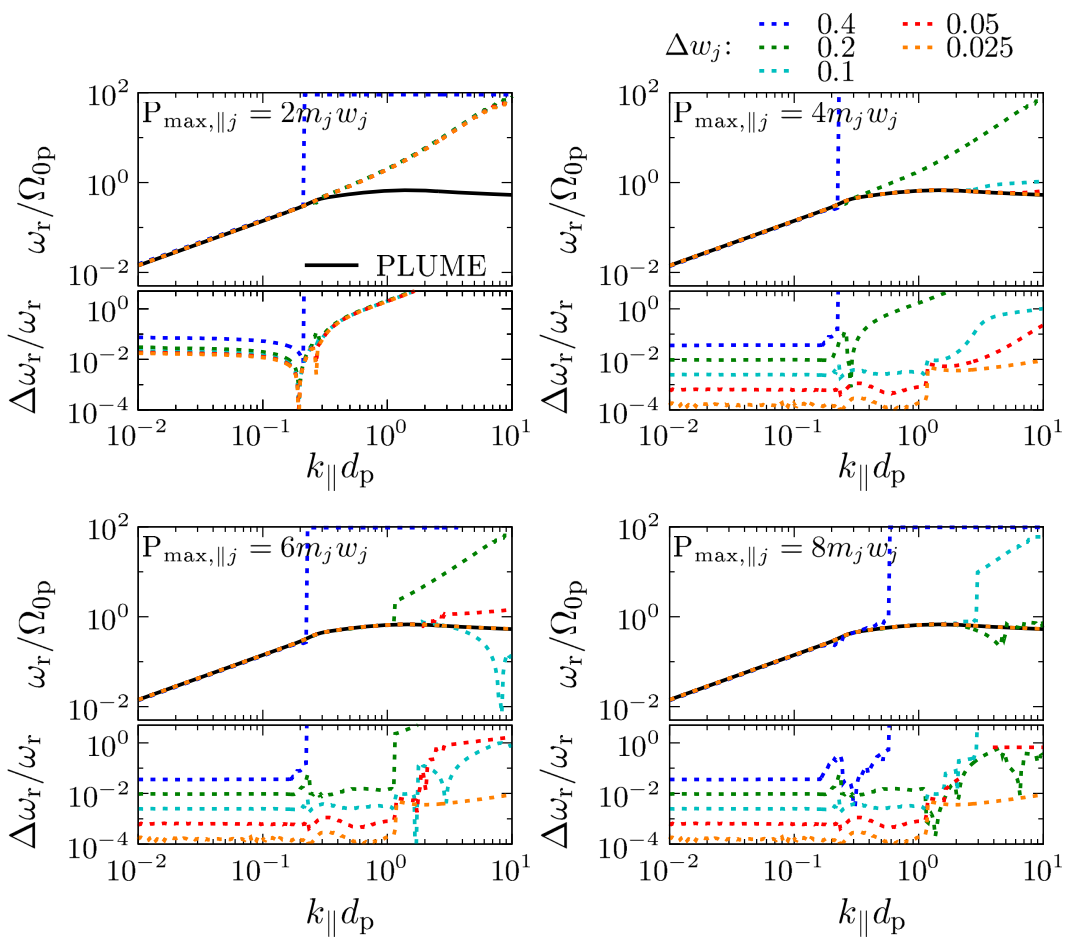

FIGURE 10. Resolution study for the real part of the frequency for the A/IC-instability solution in quasi-parallel propagation. We use a bi-Maxwellian plasma with $T_{\perp \mathrm{p}} / T_{\| \mathrm{p}}=3$. We keep $k_{\perp} d_{\mathrm{p}}=10^{-3}$ constant and scan through $k_{\|}$.

For each species, the 'fine' grid of momentum coordinates is given by $\hat{p}_{\perp, i, k}$ and $\hat{p}_{\|, i, k}$ with $i=1 \ldots N_{\perp}$ and $k=1 \ldots N_{\|}$(and correspondingly in the coordinates $\Gamma$ and $\bar{p}_{\|}$for cases with relativistic poles). The fine grid corresponds to the actual $f_{0}$ table to be used as input in ALPS. The goal of our interpolation is to find the value of the distribution function $\hat{f}_{i, k}$ on all grid points $(i, k)$. We define the vectors $\boldsymbol{w}=$ $\left(w_{1}, \ldots, w_{N_{\mathrm{c}}}\right), \boldsymbol{c}=\left(c_{1}, c_{2}, c_{3}\right), \hat{\boldsymbol{f}}_{\mathrm{c}}=\left(\hat{f}_{\mathrm{c}, 1}, \ldots, \hat{f}_{\mathrm{c}, N_{\mathrm{c}}}\right)$ and $\mathbf{0}=(0,0,0)$. We furthermore define the matrix

$$
K_{\mu, \nu}= \begin{cases}r^{2} \log (r) & \text { if } r \geqslant 1 \\ r \log \left(r^{r}\right) & \text { if } r<1\end{cases}
$$

where $r \equiv \sqrt{\left(\hat{p}_{\perp \mathrm{c}, \mu}-\hat{p}_{\perp \mathrm{c}, \nu}\right)^{2}+\left(\hat{p}_{\| \mathrm{c}, \mu}-\hat{p}_{\| \mathrm{c}, \nu}\right)^{2}}$. We also define the $\left(N_{\mathrm{c}} \times 3\right)$ matrix $\boldsymbol{P}$. Its $\mu$ th row is given by $\left(1, \hat{p}_{\perp \mathrm{c}, \mu}, \hat{p}_{\| \mathrm{c}, \mu}\right)$. The thin-plate spline interpolation requires us to solve the non-homogeneous linear system of equations

$$
\left(\begin{array}{c|c}
\boldsymbol{K}+\alpha \mathbf{1} & \boldsymbol{P} \\
\hline \boldsymbol{P}^{\top} & 0
\end{array}\right)\left(\begin{array}{c}
\boldsymbol{w} \\
\hline \boldsymbol{c}
\end{array}\right)=\left(\begin{array}{c}
\hat{\boldsymbol{f}}_{\mathrm{c}} \\
\hline \mathbf{0}
\end{array}\right)
$$

for the vectors $\boldsymbol{w}$ and $\boldsymbol{c} . \alpha$ is a user-defined smoothing parameter $(\alpha=0$ forces the fine grid to run through all points of the coarse grid), and $\mathbf{1}$ is the $\left(N_{\mathrm{c}} \times N_{\mathrm{c}}\right)$ unit 

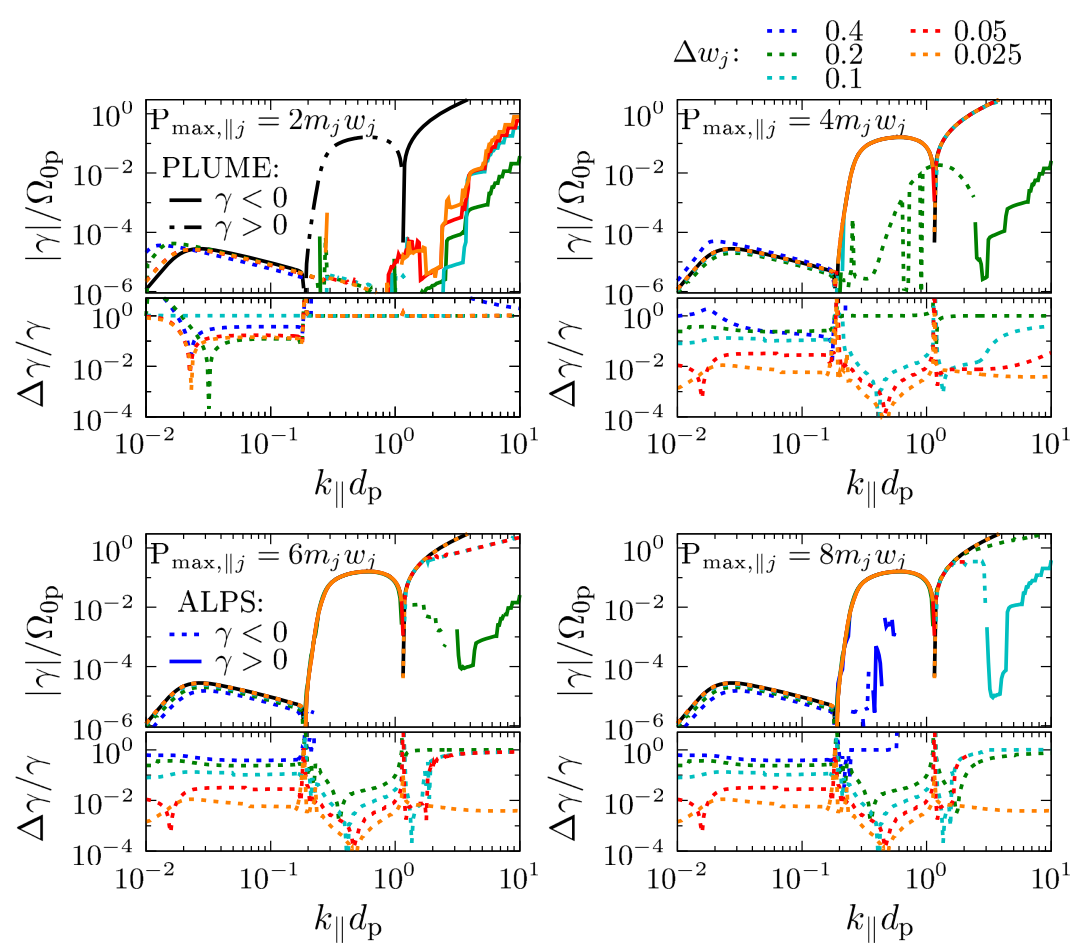

FIGURE 11. Resolution study for the imaginary part of the frequency for the A/IC-instability solution in quasi-parallel propagation. We use a bi-Maxwellian plasma with $T_{\perp \mathrm{p}} / T_{\| \mathrm{p}}=3$. We keep $k_{\perp} d_{\mathrm{p}}=10^{-3}$ constant and scan through $k_{\|}$.
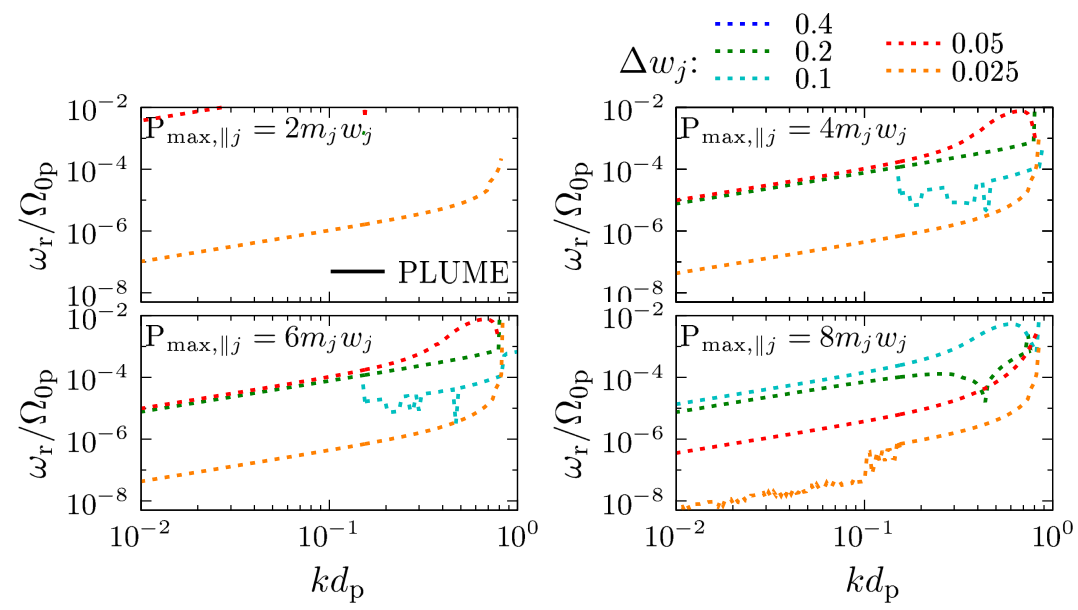

FIGURE 12. Resolution study for the real part of the frequency for the mirror-modeinstability solution. We use a bi-Maxwellian plasma with $T_{\perp \mathrm{p}} / T_{\| \mathrm{p}}=3$. We keep $\theta=75^{\circ}$ constant and scan through $|\boldsymbol{k}|$.

matrix. The interpolation is then given by

$$
\hat{f}_{i, k}=c_{1}+c_{2} \hat{p}_{\perp, i, k}+c_{3} \hat{p}_{\|, i, k}+\sum_{\mu=1}^{N_{\mathrm{c}}} w_{\mu} R_{i, k}^{\mu},
$$




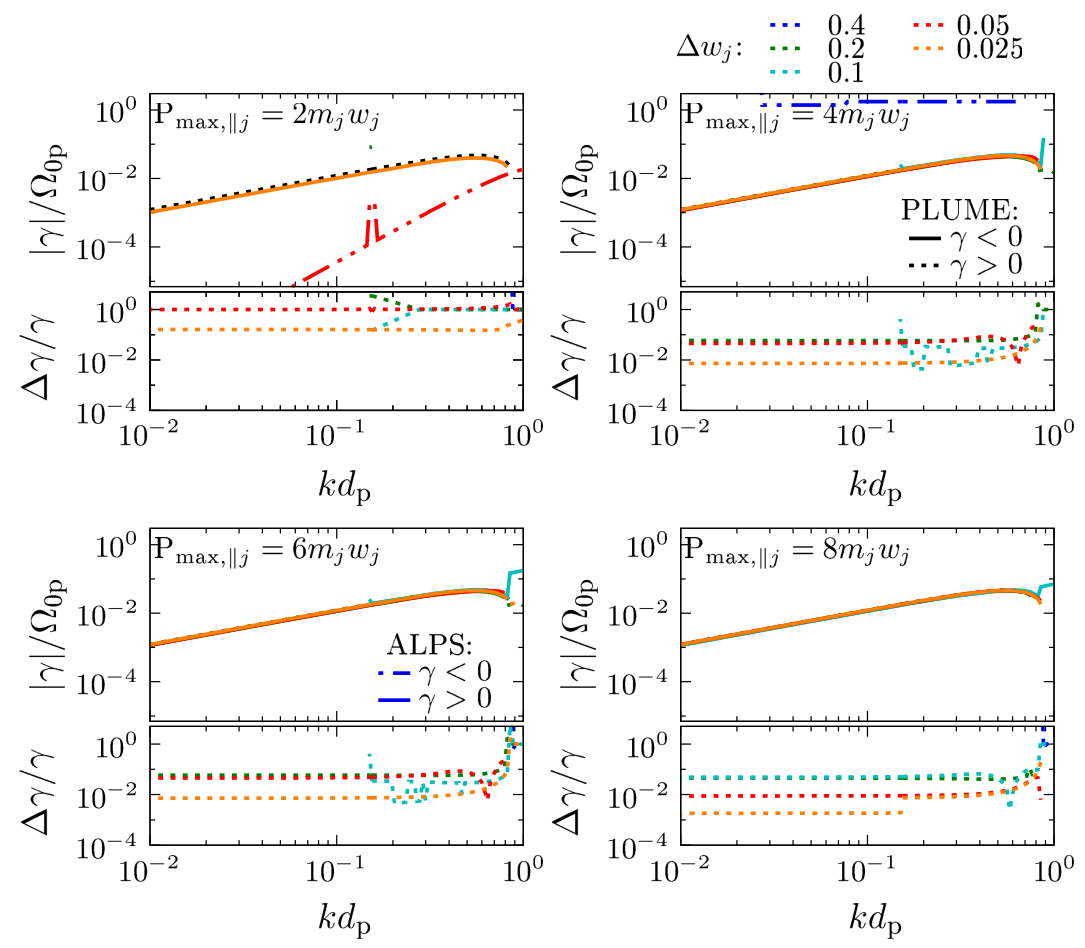

FIGURE 13. Resolution study for the imaginary part of the frequency for the mirror-modeinstability solution. We use a bi-Maxwellian plasma with $T_{\perp \mathrm{p}} / T_{\| \mathrm{p}}=3$. We keep $\theta=75^{\circ}$ constant and scan through $|\boldsymbol{k}|$.

where $R_{i, k}^{\mu} \equiv \sqrt{\left(\hat{p}_{\perp, i, k}-\hat{p}_{\perp \mathrm{c}, \mu}\right)^{2}+\left(\hat{p}_{\|, i, k}-\hat{p}_{\| \mathrm{c}, \mu}\right)^{2}}$. The numerically expensive part of the interpolation is the solution of (C2). Since $K_{11}=0$, a direct $\boldsymbol{L U}$-factorisation is not possible. Therefore, we apply a $\boldsymbol{L} \boldsymbol{U}$-factorisation algorithm with partial pivoting through row permutations until $K_{11} \neq 0$.

\section{REFERENCES}

Armstrong, T. P., Paonessa, M. T., Bell, E. V. II \& Krimigis, S. M. 1983 Voyager observations of Saturnian ion and electron phase space densities. J. Geophys. Res. 88, 8893-8904.

Astfalk, P., Görler, T. \& Jenko, F. 2015 DSHARK: a dispersion relation solver for obliquely propagating waves in bi-kappa-distributed plasmas. J. Geophys. Res. 120, 7107-7120.

Astfalk, P. \& Jenko, F. 2017 Leopard: a grid-based dispersion relation solver for arbitrary gyrotropic distributions. J. Geophys. Res. 122 (1), 89-101; $2016 J A 023522$.

Bale, S. D., Kasper, J. C., Howes, G. G., Quataert, E., Salem, C. \& Sundkvist, D. 2009 Magnetic fluctuation power near proton temperature anisotropy instability thresholds in the solar wind. Phys. Rev. Lett. 103 (21), 211101.

Buti, B. 1962 Plasma oscillations and landau damping in a relativistic gas. Phys. Fluids 5, 1-5.

Camporeale, E. \& Burgess, D. 2017 Comparison of linear modes in kinetic plasma models. J. Plasma Phys. 83 (2), 535830201.

Cannon, J. R. \& Miller, K. 1965 Some problems in numerical analytic continuation. J. Soc. Ind. Appl. Maths B 2 (1), 87. 
Cattaert, T., Hellberg, M. A. \& Mace, R. L. 2007 Oblique propagation of electromagnetic waves in a kappa-Maxwellian plasma. Phys. Plasmas 14 (8), 082111.

CAtTo, P. J. 1978 Linearized gyro-kinetics. Plasma Phys. 20, 719-722.

Chacón-Acosta, G., Dagdug, L. \& Morales-Técotl, H. A. 2010 Manifestly covariant Jüttner distribution and equipartition theorem. Phys. Rev. E 81 (2), 021126.

Christon, S. P., Mitchell, D. G., Williams, D. J., Frank, L. A., Huang, C. Y. \& Eastman, T. E. 1988 Energy spectra of plasma sheet ions and electrons from about $50 \mathrm{eV} / \mathrm{e}$ to about $1 \mathrm{MeV}$ during plamsa temperature transitions. J. Geophys. Res. 93, 2562-2572.

Davidson, R. C. \& Ogden, J. M. 1975 Electromagnetic ion cyclotron instability driven by ion energy anisotropy in high-beta plasmas. Phys. Fluids 18, 1045-1050.

DaVis, P. J. \& Rabinowitz, P. 1984 Methods of Numerical Integration, 2nd edn. Academic Press.

Donato, G. \& Belongie, S. 2002 Approximate thin plate spline mappings. In Proceedings of the 7th European Conference on Computer Vision-Part III, pp. 21-31. Springer.

Drummond, W. E. \& Rosenbluth, M. N. 1963 Cyclotron radiation from a hot plasma. Phys. Fluids 6, 276-283.

Dum, C. T., MARSCh, E. \& Pilipp, W. 1980 Determination of wave growth from measured distribution functions and transport theory. J. Plasma Phys. 23, 91-113.

Egedal, J., Daughton, W. \& Le, A. 2012 Large-scale electron acceleration by parallel electric fields during magnetic reconnection. Nature 8, 321-324.

Egedal, J., Le, A. \& Daughton, W. 2013 A review of pressure anisotropy caused by electron trapping in collisionless plasma, and its implications for magnetic reconnection. Phys. Plasmas 20 (6), 061201.

Eviatar, A. \& Schulz, M. 1970 Ion-temperature anisotropies and the structure of the solar wind. Planet. Space Sci. 18, 321-332.

Feldman, W. C., Asbridge, J. R., Bame, S. J., Montgomery, M. D. \& Gary, S. P. 1975 Solar wind electrons. J. Geophys. Res. 80, 4181-4196.

Fu, C.-L., Zhang, Y.-X., Cheng, H. \& MA, Y.-J. 2012 Numerical analytic continuation on bounded domains. Engng Anal. Bound. Elem. 36, 493.

Fujiwara, H., Imai, H., TAKeuchi, T. \& Iso, Y. 2007 Numerical treatment of analytic continuation with multiple-precision arithmetic. Hokkaido Math. J. 36, 837.

GAELzer, R. \& Ziebell, L. F. 2016 Obliquely propagating electromagnetic waves in magnetized kappa plasmas. Phys. Plasmas 23 (2), 022110.

Gaelzer, R., Ziebell, L. F. \& Meneses, A. R. 2016 The general dielectric tensor for bi-kappa magnetized plasmas. Phys. Plasmas 23 (6), 062108.

GAFFEY, J. D. JR. $1976 a$ Energetic ion distribution resulting from neutral beam injection in tokamaks. J. Plasma Phys. 16, 149-169.

GAffey, J. D. JR. 1976b Instability of energetic ion beam injection in tokamaks. J. Plasma Phys. 16, 171-179.

Galvaõ, R. A., Ziebell, L. F., Gaelzer, R. \& De Juli, M. C. 2012 Alfvén waves in dusty plasmas with plasma particles described by anisotropic kappa distributions. Phys. Plasmas 19 (12), 123705.

Gary, S. P. 1993 Theory of Space Plasma Microinstabilities. Cambridge University Press.

Gosling, J. T. 2007 Observations of magnetic reconnection in the turbulent high-speed solar wind. Astrophys. J. Lett. 671, L73-L76.

Gosling, J. T., Asbridge, J. R., Bame, S. J., Feldman, W. C., Zwickl, R. D., Paschmann, G., SCKOPKE, N. \& HYNDS, R. J. 1981 Interplanetary ions during an energetic storm particle event - the distribution function from solar wind thermal energies to $1.6 \mathrm{MeV}$. J. Geophys. Res. 86, 547-554.

Gosling, J. T., Eriksson, S., Phan, T. D., Larson, D. E., Skoug, R. M. \& McComas, D. J. 2007 Direct evidence for prolonged magnetic reconnection at a continuous x-line within the heliospheric current sheet. Geophys. Res. Lett. 34, 6102.

HARRIs, E. G. 1961 Plasma instabilities associated with anisotropic velocity distributions. J. Nucl. Energy 2, 138-145. 
HeIdBrink, W. W. \& SADLER, G. J. 1994 REVIEW PAPER: the behaviour of fast ions in tokamak experiments. Nucl. Fusion 34, 535-615.

Hellberg, M., Mace, R. \& Cattaert, T. 2005 Effects of superthermal particles on waves in magnetized space plasmas. Space Sci. Rev. 121, 127-139.

Hellinger, P., Trávníček, P., Kasper, J. C. \& Lazarus, A. J. 2006 Solar wind proton temperature anisotropy: linear theory and WIND/SWE observations. Geophys. Res. Lett. 33, 9101.

Hellinger, P. \& Trávníček, P. M. 2011 Proton core-beam system in the expanding solar wind: hybrid simulations. J. Geophys. Res. 116, 11101.

Hellinger, P. \& Trávníček, P. M. 2013 Protons and alpha particles in the expanding solar wind: hybrid simulations. J. Geophys. Res. 118, 5421-5430.

Howes, G. G., Cowley, S. C., Dorland, W., Hammett, G. W., Quataert, E. \& Schekochinin, A. A. 2006 Astrophysical gyrokinetics: basic equations and linear theory. Astrophys. J. 651, 590-614.

Hundhausen, A. J. 1970 Composition and dynamics of the solar wind plasma. Rev. Geophys. Space Phys. 8, 729-811.

IsEnBERG, P. A. 2012 A self-consistent marginally stable state for parallel ion cyclotron waves. Phys. Plasmas 19 (3), 032116.

JÜtTneR, F. 1911 Das Maxwellsche Gesetz der Geschwindigkeitsverteilung in der Relativtheorie. Ann. Phys. 339, 856-882.

Klein, K. G., Alterman, B. L., Stevens, M. L., Vech, D. \& Kasper, J. C. 2018 Majority of solar wind intervals support ion-driven instabilities. Phys. Rev. Lett. 120, 205102.

Klein, K. G. \& Howes, G. G. 2015 Predicted impacts of proton temperature anisotropy on solar wind turbulence. Phys. Plasmas 22 (3), 032903.

Klein, K. G., Howes, G. G., Tenbarge, J. M., Bale, S. D., Chen, C. H. K. \& Salem, C. S. 2012 Using synthetic spacecraft data to interpret compressible fluctuations in solar wind turbulence. Astrophys. J. 755, 159.

Klein, K. G., Kasper, J. C., Korreck, K. E. \& Stevens, M. L. 2017 Applying Nyquist's method for stability determination to solar wind observations. J. Geophys. Res. 122, 9815-9823.

Kranich, S. 2014 Computational analytic continuation. Preprint, arXiv:1403.2858.

Kunz, M. W., Schekochinin, A. A., Chen, C. H. K., Abel, I. G. \& Cowley, S. C. 2015 Inertial-range kinetic turbulence in pressure-anisotropic astrophysical plasmas. J. Plasma Phys. 81 (5), 325810501.

Landau, L. D. 1946 On the vibrations of the electronic plasma. J. Phys. (USSR) 10, 25-34; [Zh. Eksp. Teor. Fiz. 16,574 (1946)].

LAZAR, M. \& PoedTs, S. 2014 Instability of the parallel electromagnetic modes in Kappa distributed plasmas - II. Electromagnetic ion-cyclotron modes. Mon. Not. R. Astron. Soc. 437, 641-648.

Lazar, M., Poedts, S. \& Schlickeiser, R. 2011 Proton firehose instability in bi-Kappa distributed plasmas. Astron. Astrophys. 534, A116.

LAZAR, M. \& SCHLICKeISER, R. 2006 Relativistic kinetic dispersion theory of linear parallel waves in magnetized plasmas with isotropic thermal distributions. New J. Phys. 8, 66.

Lazar, M. \& Poedts, S. 2009 Firehose instability in space plasmas with bi-kappa distributions. Astron. Astrophys. 494, 311-315.

LERCHE, I. 1967 Unstable magnetosonic waves in a relativistic plasma. Astrophys. J. 147, 689.

LERCHE, I. 1968 Supra-luminous waves and the power spectrum of an isotropic, homogeneous plasma. Phys. Fluids 11, 413-422.

LEUbNeR, M. P. 1978 Influence of non-bi-Maxwellian distribution function of solar wind protons on the ion cyclotron instability. J. Geophys. Res. 83, 3900-3902.

LEVEnBerG, K. 1944 A method for the solution of certain non-linear problems in least squares. Q. Appl. Maths 2 (2), 164-168.

Lifshitz, E. M. \& Pitaevskit, L. P. 1981 Physical Kinetics. Pergamon Press.

Longman, I. M. 1958 On the numerical evaluation of cauchy principal values of integrals. Math. Tables Other Aids Comput. 12 (63), 205. 
López, R. A., Moya, P. S., Muñoz, V., Viñas, A. F. \& Valdivia, J. A. 2014 Kinetic transverse dispersion relation for relativistic magnetized electron-positron plasmas with Maxwell-Jüttner velocity distribution functions. Phys. Plasmas 21 (9), 092107.

López, R. A., Moya, P. S., Navarro, R. E., Araneda, J. A., Muñoz, V., Viñas, A. F. \& VAlDiviA, A. J. 2016 Relativistic cyclotron instability in anisotropic plasmas. Astrophys. $J$. 832, 36.

Lui, A. T. Y. \& KRIMigis, S. M. 1981 Earthward transport of energetic protons in the earth's plasma sheet. Geophys. Res. Lett. 8, 527-530.

Lui, A. T. Y. \& Krimigis, S. M. 1983 Energetic ion beam in the earth's magnetotail lobe. Geophys. Res. Lett. 10, 13-16.

MACE, R. L. \& SydorA, R. D. 2010 Parallel whistler instability in a plasma with an anisotropic bi-kappa distribution. J. Geophys. Res. 115, 7206.

MARquardT, D. W. 1963 An algorithm for least-squares estimation of nonlinear parameters. J. Soc. Ind. Appl. Maths 11 (2), 431-441.

MARSCH, E. 2006 Kinetic physics of the solar corona and solar wind. Living Rev. Solar Phys. 3, 1.

Marsch, E., Rosenbauer, H., Schwenn, R., Muehlhaeuser, K.-H. \& Neubauer, F. M. $1982 a$ Solar wind helium ions - Observations of the HELIOS solar probes between 0.3 and 1 AU. J. Geophys. Res. 87, 35-51.

Marsch, E., Schwenn, R., Rosenbauer, H., Muehlhaeuser, K.-H., Pilipp, W. \& Neubauer, F. M. $1982 b$ Solar wind protons - three-dimensional velocity distributions and derived plasma parameters measured between 0.3 and 1 AU. J. Geophys. Res. 87, 52-72.

MARSCH, E. \& TU, C.-Y. 2001 Evidence for pitch angle diffusion of solar wind protons in resonance with cyclotron waves. J. Geophys. Res. 106, 8357-8362.

Maruca, B. A., Kasper, J. C. \& GARY, S. P. 2012 Instability-driven limits on helium temperature anisotropy in the solar wind: observations and linear vlasov analysis. Astrophys. J. 748, 137.

Matsuda, Y. \& SMith, G. R. 1992 A microinstability code for a uniform magnetized plasma with an arbitrary distribution function. J. Comput. Phys. 100, 229-235.

Phan, T. D., Gosling, J. T., Davis, M. S., Skoug, R. M., Øieroset, M., Lin, R. P., Lepping, R. P., McComas, D. J., Smith, C. W., Reme, H. et al. 2006 A magnetic reconnection $\mathrm{X}$-line extending more than 390 Earth radii in the solar wind. Nature 439, 175-178.

Pilipp, W. G., Muehlhaeuser, K.-H., Miggenrieder, H., Montgomery, M. D. \& Rosenbauer, H. 1987a Characteristics of electron velocity distribution functions in the solar wind derived from the HELIOS plasma experiment. J. Geophys. Res. 92, 1075-1092.

Pilipp, W. G., Muehlhaeuser, K.-H., Miggenrieder, H., Rosenbauer, H. \& Schwenn, R. $1987 b$ Variations of electron distribution functions in the solar wind. J. Geophys. Res. 92, 1103-1118.

Powell, M. J. D. 1994 Some algorithms for thin-plate spline interpolation to functions of two variables. In Advances in Computational Mathematics, New Delhi, India (ed. H. P. Dikshit \& C. A. Micchelli), pp. 303-319. World Scientific.

Press, W. H., Teukolsky, S. A., Vetterling, W. T. \& Flannery, B. P. 1992 Numerical Recipes in FORTRAN. The Art of Scientific Computing, 2nd edn. Cambridge University Press.

Pulupa, M., Salem, C. S., Horaites, K. I. \& Bale, S. 2011 Solar wind electron microphysics: results from a Wind electron database. In AGU Fall Meeting Abstracts, p. B2034.

QUATAERT, E. 1998 Particle heating by alfvenic turbulence in hot accretion flows. Astrophys. J. 500, 978.

ReICHEL, L. 1986 Numerical methods for analytic continuation and mesh generation. Constr. Approx. 2, 23.

Reisenfeld, D. B., Gary, S. P., Gosling, J. T., Steinberg, J. T., McComas, D. J., Goldstein, B. E. \& Neugebauer, M. 2001 Helium energetics in the high-latitude solar wind: Ulysses observations. J. Geophys. Res. 106, 5693-5708.

Robinson, P. A. 1990 Systematic methods for calculation of the dielectric properties of arbitrary plasmas. J. Comput. Phys. 88, 381-392. 
ROENNMARK, K. 1982 Waves in homogeneous, anisotropic multicomponent plasmas (WHAMP). Report No. 179, ISSN: 0347-6406. Kiruna, Sweden: Kiruna Geophysical Institute.

Rudakov, L. I. \& SAgDeEv, R. Z. 1961 On the instability of a nonuniform rarefied plasma in a strong magnetic field. Sov. Phys. Dokl. 6, 415.

Salem, C. S., Pulupa, M., Verscharen, D., Bale, S. D. \& Chandran, B. D. 2013 Electron temperature anisotropies in the solar wind: properties, regulation and constraints. In $A G U$ Fall Meeting Abstracts, p. B2104.

SChwartz, S. J. 1980 Plasma instabilities in the solar wind - a theoretical review. Rev. Geophys. Space Phys. 18, 313-336.

Seki, M., Saigusa, M., Nemoto, M., Kusama, K., Tobita, T., Kuriyama, M. \& Uehara, K. 1989 Observation of ion-cyclotron-wave instability caused by perpendicular neutral beam injection in the JT-60 tokamak. Phys. Rev. Lett. 62, 1989-1992.

ShKAROFSKY, I. P. 1966 Dielectric tensor in Vlasov plasmas near cyclotron harmonics. Phys. Fluids 9, 561-570.

Southwood, D. J. \& Kivelson, M. G. 1993 Mirror instability. I - physical mechanism of linear instability. J. Geophys. Res. 98, 9181-9187.

STIX, T. H. 1975 Fast-wave heating of a two-component plasma. Nucl. Fusion 15, 737-754.

StIX, T. H. 1992 Waves in Plasmas. American Institute of Physics.

Štverák, Š., Maksimovic, M., Trávníček, P. M., Marsch, E., Fazakerley, A. N. \& Scime, E. E. 2009 Radial evolution of nonthermal electron populations in the low-latitude solar wind: helios, cluster, and ulysses observations. J. Geophys. Res. 114, A05104.

Summers, D. \& Thorne, R. M. 1991 The modified plasma dispersion function. Phys. Fluids B 3, $1835-1847$.

Summers, D., Xue, S. \& Thorne, R. M. 1994 Calculation of the dielectric tensor for a generalized Lorentzian (kappa) distribution function. Phys. Plasmas 1, 2012-2025.

Swanson, D. G. 2002 Exact and moderately relativistic plasma dispersion functions. Plasma Phys. Control. Fusion 44, 1329-1347.

TAJIRI, M. 1967 Propagation of hydromagnetic waves in collisionless plasma. II. Kinetic Approach. J. Phys. Soc. Japan 22, 1482.

TANG, W. M. 1978 Microinstability theory in tokamaks. Nucl. Fusion 18, 1089-1160.

TRubnikov, B. A. 1959 Electromagnetic waves in a relativistic plasma in the presence of a magnetic field. In Plasma Physics and the Problem of Controlled Thermonuclear Reactions (ed. M. A. Leontovich), vol. 3, p. 122. Pergamon Press.

VASYliunas, V. M. 1968 A survey of low-energy electrons in the evening sector of the magnetosphere with OGO 1 and OGO 3. J. Geophys. Res. 73, 2839-2884.

Verscharen, D., Bourouaine, S. \& Chandran, B. D. G. 2013 Instabilities driven by the drift and temperature anisotropy of alpha particles in the solar wind. Astrophys. J. 773, 163.

Verscharen, D. \& Chandran, B. D. G. 2013 The dispersion relations and instability thresholds of oblique plasma modes in the presence of an ion beam. Astrophys. J. 764, 88.

Verscharen, D. \& ChAndRAn, B. D. G. 2018 NHDS: the new hampshire dispersion relation solver. Res. Notes AAS 2, 13.

Verscharen, D., Chandran, B. D. G., Klein, K. G. \& Quataert, E. 2016 Collisionless isotropization of the solar-wind protons by compressive fluctuations and plasma instabilities. Astrophys. J. 831, 128.

Verscharen, D., Chen, C. H. K. \& WiCKs, R. T. 2017 On kinetic slow modes, fluid slow modes, and pressure-balanced structures in the solar wind. Astrophys. J. 840, 106.

Weideman, J. A. C. 1995 Computing the Hilbert transform on the real line. Math. Comput. 64, $745-762$.

Williams, D. J., Mitchell, D. G. \& Christon, S. P. 1988 Implications of large flow velocity signatures in nearly isotropic ion distributions. Geophys. Res. Lett. 15, 303-306.

XIE, H.-S. 2013 Generalized plasma dispersion function: one-solve-all treatment, visualizations, and application to Landau damping. Phys. Plasmas 20 (9), 092125. 
Xue, S., Thorne, R. M. \& Summers, D. 1993 Electromagnetic ion-cyclotron instability in space plasmas. J. Geophys. Res. 98, 17475-17484.

Xue, S., Thorne, R. M. \& Summers, D. 1996 Excitation of magnetosonic waves in the undisturbed solar wind. Geophys. Res. Lett. 23, 2557-2560.

Yoon, P. H., Seough, J. J., Khim, K. K., Kim, H., Kwon, H.-J., Park, J., Parkh, S. \& PARK, K. S. 2010 Analytic model of electromagnetic ion-cyclotron anisotropy instability. Phys. Plasmas 17 (8), 082111.

ZHANG, Z.-Q. \& MA, Y.-J. 2013 A modified kernel method for numerical analytic continuation. Inverse Prob. Sci. Engng 21 (5), 840. 